\title{
Theoretical analysis of two-step holographic recording with high-intensity pulses
}

\author{
Ali Adibi, ${ }^{*}$ Karsten Buse, ${ }^{\dagger}$ and Demetri Psaltis \\ Department of Electrical Engineering, California Institute of Technology, Pasadena, California 91125
}

(Received 14 July 2000; published 17 January 2001)

\begin{abstract}
We develop a full numerical as well as an approximate analytic solution for two-step holographic recording with high intensity pulses in $\mathrm{LiNbO}_{3}: \mathrm{Fe}$ crystals. We find the unknown material parameters by fitting the numerical solution to the experimental results. The two important parameters that were unknown so far and found in this work are the bulk photovoltaic coefficient and absorption cross section for the excitation of the electrons from small polarons in $\mathrm{LiNbO}_{3}$ with infrared light. We show that the approximate analytic solution agrees very well with the numerical solution (as well as the experimental results) for most practical applications. We use the analytic solution to explain the experimental observations that were not understood before.

DOI: 10.1103/PhysRevA.63.023813

PACS number(s): 42.65.Hw, 42.40.Ht, 42.70.Ln, 42.40.Pa
\end{abstract}

\section{INTRODUCTION}

Photorefractive crystals are excellent candidates for volume holographic storage [1-3]. A major obstacle in making practical read/write holographic memory systems has been nonpersistence (or destructive readout) of the stored information. Thermal fixing [4] and electrical fixing [5] are the two major nonoptical methods for obtaining persistence. However, they require heating the sample or applying large electric fields. All-optical methods for persistent holographic recording include frequency-difference holograms [6], readout with wave-vector spectra [7], and gated recording [8,9]. Among all the methods proposed, gated recording is the most promising one for obtaining persistent read/write holographic memories.

Gated holographic recording relies on the existence of two sets of traps (shallower and deeper traps) with energy levels in the band gap of the recording crystal. These traps can be due to doping by impurities (for example, $\mathrm{LiNbO}_{3}: \mathrm{Fe}: \mathrm{Mn}$ crystals [9]) or (at least one set of traps) can be due to intrinsic traps [8] (polarons, bipolarons, etc.). We refer to recording using the former as "two-center recording" and to that using the latter as "two-step recording," since intrinsic defects can occur in a very high concentration enabling direct charge transfer between the shallower and the deeper traps. Recording is performed by the simultaneous presence of a sensitizing (or gating) beam of shorter wavelength (higher photon energy) and two recording beams of longer wavelength (lower photon energy). Electrons are initially in the deeper traps (shallower traps are initially empty). Sensitizing light causes the electron transfer from the deeper traps to the shallower traps. The hologram is recorded by the recording beams using the electrons from the shallower traps. The final hologram is imprinted in the deeper traps, and persists against readout with the light of longer wavelength (same as recording wavelength). In this paper, we mainly consider two-step holographic recording in

\footnotetext{
*Present address: Georgia Institute of Technology, School of Electrical and Computer Engineering, Atlanta, GA 30332.

${ }^{\dagger}$ Present address: University of Bonn, Physical Institute, Wegelestr. 8, D-53115 Bonn, Germany.
}

$\mathrm{LiNbO}_{3}: \mathrm{Fe}$ crystals with green pulses for sensitization and infrared pulses for recording.

Most of the initial two-step holographic recording experiments were performed with high intensity pulses in congruent $\mathrm{LiNbO}_{3}[8,10-12]$ and $\mathrm{LiTaO}_{3}$ [13] crystals. More recently, two-step recording experiments using stoichiometric $\mathrm{LiNbO}_{3}$ crystals with $\mathrm{cw}$ light were reported [14-18]. The shallower traps in two-step recording in $\mathrm{LiNbO}_{3}: \mathrm{Fe}$ and $\mathrm{LiNbO}_{3}: \mathrm{Cu}$ are due to the small $\mathrm{Nb}_{\mathrm{Li}}$ polarons caused by niobium on the lithium site [19,20].

A two-center model for two-step recording with the light of only one wavelength (same wavelength for sensitization and recording) was proposed [21]. However, a theoretical analysis of the two-step persistent storage in $\mathrm{LiNbO}_{3}: \mathrm{Fe}$ is still missing. The question is whether the iron-polaron model can describe quantitatively the obtained experimental results using the charge transport parameters known for $\mathrm{LiNbO}_{3}$ from the literature. The aim is to achieve a model and a parameter set that explains all photorefractive features of congruent $\mathrm{LiNbO}_{3}$, at low and high light intensities, for oneand two-step recording. A full theoretical description and understanding of the processes is highly desired, because then the optimum performance of the material and the conditions to achieve this performance can be predicted. Furthermore, there are several experimental observations that have not been explained yet. Having a reliable model is very helpful in understanding the physical mechanisms responsible for two-step recording and the explanation of the experimental observations. First general attempts of a formal analysis of the processes involved in different two-step recording schemes were performed only for materials with negligible bulk photovoltaic effect [22].

In this paper, we present a full theoretical analysis of two-step holographic recording in $\mathrm{LiNbO}_{3}: \mathrm{Fe}$ crystals with high intensity green pulses for sensitization, and infrared pulses for recording. We start with the two-center model and first develop a full numerical solution of the governing equations without any approximation. We compare the numerical solution with the experimental results to compute two unknown parameters of the shallow polaron levels in congruent $\mathrm{LiNbO}_{3}$ at infrared. Since the variations of the holographic recording properties during one short pulse are small, we expand all variables in the governing equations by the first few terms of their Taylor expansion to develop an analytic 
solution to the governing equations within one pulse width. We then use the appropriate initial conditions at the beginning and at the end of each pulse to obtain analytic formulas for major holographic recording parameters at all time.

After finding the analytic formulas for the saturation space-charge field and the recording time constant of a hologram, we use some approximations to simplify the equations for explaining all experimental observations (including those that were not explained before) based on simple physical principles. For normal recording in $\mathrm{LiNbO}_{3}$ (one set of traps, one light wavelength), the formulas for the saturation space-charge field $\left(\left.E\right|_{\text {saturation }}\right)$ and recording time constant $\left(\tau_{r}\right)$,

$$
\begin{aligned}
\left.E\right|_{\text {saturation }} & =-\frac{\kappa_{D} N_{D}^{-} I_{R}}{e \mu n_{0}}, \\
\frac{1}{\tau_{r}} & =\frac{e \mu n_{0}}{\epsilon \epsilon_{0}},
\end{aligned}
$$

are well known. In these equations, $\kappa_{D}, \epsilon \epsilon_{0}, \mu$, and $e$ are the bulk photovoltaic constant of the deep traps (for example, $\mathrm{Fe}$ in $\mathrm{LiNbO}_{3}: \mathrm{Fe}$ ), permittivity of $\mathrm{LiNbO}_{3}$, electron mobility in the conduction band, and electronic charge, respectively. Furthermore, $N_{D}^{-}, n_{0}$, and $I_{R}$ are the average (or dc) electron concentration in the deep traps, average electron concentration in the conduction band, and the amplitude of the space-varying part of the recording intensity, respectively. Our theoretical analysis shows that we can also use Eqs. (1) and (2) for two-step recording if we simply replace $N_{D}^{-}$and $n_{0}$ by $N_{X 0 \text {,ave }}^{-}$and $n_{0 \text {,ave }}$ (the values of the dc components of the electron concentrations in the shallower traps and in the conduction band, respectively, time-averaged within one pulse width). This is a major step in understanding the dominant processes in two-step holographic recording, and explaining all the experimental results.

\section{EXPERIMENTS}

Melt-doped single domain $\mathrm{LiNbO}_{3}: \mathrm{Fe}$ samples grown by the Czochralski technique are investigated. The total $\mathrm{Fe}$ concentration $c_{\mathrm{Fe}}$ of the samples is determined by x-ray fluorescence and atomic absorption spectroscopy. The samples contain typically between 370 and $1070 \mathrm{~mol} \mathrm{ppm} \mathrm{Fe.} \mathrm{The}$ uncertainties of the determined $c_{\mathrm{Fe}}$ values are about $\pm 15 \%$.

The valence states of the $\mathrm{Fe}$ ions are varied by suitable annealing treatments [23]. Heating in pure oxygen atmosphere, e.g., to a temperature of $1000^{\circ} \mathrm{C}$, tends to oxidize the ions to $\mathrm{Fe}^{3+}$, whereas heating in argon atmosphere or vacuum (low oxygen partial pressure) yields a reduction of the ions to $\mathrm{Fe}^{2+}$.

Determination of the concentrations $c_{\mathrm{Fe}^{2}+}$ and $c_{\mathrm{Fe}^{3+}}$ is based on Mössbauer experiments [23]. From the comparison of the Mössbauer results with optical absorption measurements, the oscillator strengths of the bands are calculated [24]. The absorption coefficient at $477 \mathrm{~nm}$ for ordinarily polarized light, determined by a Cary $17 \mathrm{D}$ spectrometer, yields $c_{\mathrm{Fe}^{2+}}$. Then, $c_{\mathrm{Fe}^{3+}}$ can be determined because the entire $\mathrm{Fe}$ concentration of the crystal is known, and the Mössbauer results clearly demonstrate that only $\mathrm{Fe}^{2+}$ and $\mathrm{Fe}^{3+}$ states of

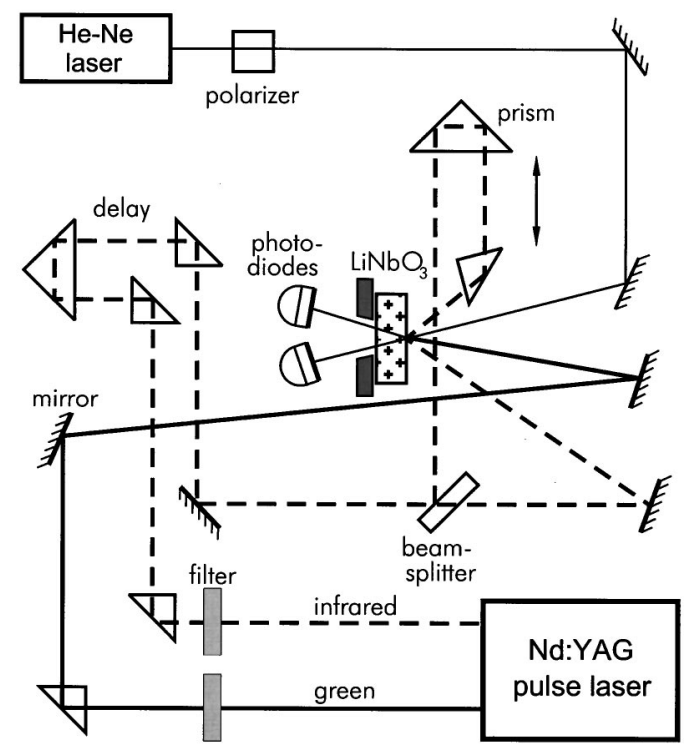

FIG. 1. Schematic drawing of the experimental setup for twostep holographic recording.

$\mathrm{Fe}$ ions are present in $\mathrm{LiNbO}_{3}: \mathrm{Fe}$ crystals. Typically ratios of $c_{\mathrm{Fe}^{2+}} / c_{\mathrm{Fe}^{3+}}$ in the range from 0.01 to 1 can be adjusted easily.

Figure 1 shows a schematic illustration of the holographic setup. A $Q$-switched Nd:YAG laser with a frequency doubler produces simultaneously infrared $(\lambda=1064 \mathrm{~nm}$, pulse duration $25 \mathrm{~ns})$ and green $(\lambda=532 \mathrm{~nm}$, pulse duration $15 \mathrm{~ns})$ ordinarily polarized $\mathrm{TEM}_{00}$ light pulses. The repetition rate of the system used is only about $0.1 \mathrm{~Hz}$. A dielectric beam splitter separates the infrared and green light. An additional beam splitter divides the infrared light into two coherent beams of equal intensity. These beams enter the crystal symmetrically in a plane containing the crystal's $\vec{c}$ axis. The green pulse enters the sample simultaneously or with a delay of up to $100 \mathrm{~ns}$ achieved by an optical path difference.

Holographic readout is performed by low intensity ordinarily polarized continuous-wave $\mathrm{HeNe}$ laser light $(\lambda$ $=633 \mathrm{~nm}$ ) entering the crystal under the Bragg angle. Photodiodes behind the sample detect transmitted and diffracted light intensities. The diffraction efficiency is defined as the ratio of the intensities of the diffracted and total transmitted light. From Kogelnik's formula [25], we then calculate the refractive index changes. The intersection angle of the infrared pulses and the light wavelength determine the fringe spacing $\Lambda$. This $\Lambda$ value is in the employed transmission geometry typically about 1 to $2 \mu \mathrm{m}$. Neutral density filters provide variations of infrared and green light intensities.

Figure 2 illustrates a typical hologram writing and erasing cycle. The time scale corresponds to the exposure time of the green $(\lambda=532 \mathrm{~nm})$ light. The circles represent experimental data and the solid lines are exponential fits taking into account absorption effects [26]. Typical total infrared and green light intensities are $I_{1064}=250 \mathrm{GW} \mathrm{m}^{-2}$ and $I_{532}$ $=110 \mathrm{GW} \mathrm{m}^{-2}$.

\section{TWO-CENTER MODEL}

The two-center charge transport model for $\mathrm{LiNbO}_{3}: \mathrm{Fe}$ was introduced in 1993 by Jermann and Otten [21]. Figure 3 


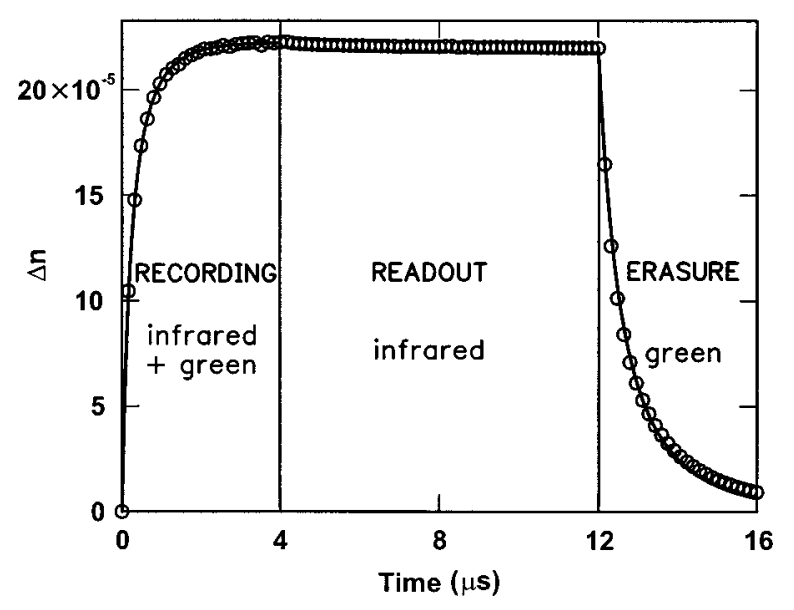

FIG. 2. Refractive-index amplitude $\Delta n$ of a holographic grating during a typical writing and erasing cycle. The circles illustrate the experimental data and the solid lines are monoexponential fits taking into account absorption effects [26]. The time scale corresponds to the exposure time of the green $(\lambda=532 \mathrm{~nm})$ light. During the first $4-\mu$ s infrared and green light (wavelengths: $\lambda=1064 \mathrm{~nm}, \lambda$ $=532 \mathrm{~nm}$; intensities: $I_{1064}=250 \mathrm{GWm}^{-2}, I_{532}=110 \mathrm{GWm}^{-2}$ ) are simultaneously present. The next $8-\mu$ s readout of the hologram with one of the infrared writing beams $\left(I_{1064}=125 \mathrm{GWm}^{-2}, I_{532}=0\right)$ is performed. After this second step, the hologram is erased by green light $\left(I_{1064}=0, I_{532}=110 \mathrm{GWm}^{-2}\right)$.

shows the band diagram of a $\mathrm{LiNbO}_{3}: \mathrm{Fe}$ crystal. Electrons can be excited from $\mathrm{Fe}^{2+}$ by light either into the conduction band or into $\mathrm{Nb}_{\mathrm{Li}}^{5+}$ forming $\mathrm{Nb}_{\mathrm{Li}}^{4+}$. Direct excitation into $\mathrm{Nb}_{\mathrm{Li}}$ requires that there are always some $\mathrm{Nb}_{\mathrm{Li}}$ centers close to each $\mathrm{Fe}^{2+}$. This is the case, because $\mathrm{Nb}_{\mathrm{Li}}$ is an intrinsic defect that occurs in a very high concentration $[19,20]$. The electrons in the shallower $\mathrm{Nb}_{\mathrm{Li}}^{4+}$ traps can be excited to the conduction band by light or thermally. Otherwise, they recombine directly with the iron ions where they come from. The conduction-band electrons can recombine either with $\mathrm{Fe}^{3+}$ or with $\mathrm{Nb}_{\mathrm{Li}}^{5+}$. The iron level is "deep" and the polaron level is often called "shallow," although these words have a different meaning in semiconductor physics, where shallow levels are characterized by a strong thermal generation rate.

Green light (wavelength $532 \mathrm{~nm}$ ) has sufficient photon

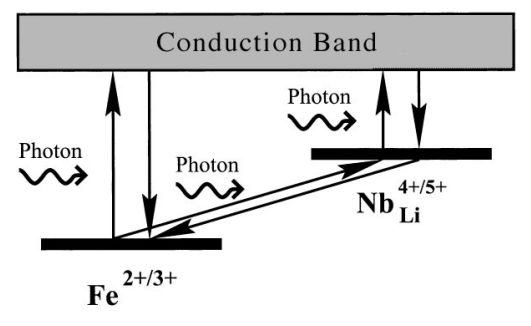

Valence Band

FIG. 3. Band diagram of the charge transport situation in congruent iron-doped lithium niobate $\left(\mathrm{LiNbO}_{3}\right)$. The arrows indicate excitation and recombination of electrons. A detailed description is given in the text. energy to excite electrons from $\mathrm{Fe}^{2+}$ either into the conduction band or into the secondary centers, or from $\mathrm{Nb}_{\mathrm{Li}}^{4+}$ into the conduction band. Infrared light (wavelength $1064 \mathrm{~nm}$ ), however, has a smaller photon energy that is sufficient to excite electrons from $\mathrm{Nb}_{\mathrm{Li}}^{4+}$ into the conduction band, only.

Excitation and recombination of the electrons can be described by the rate equations [21]

$$
\begin{aligned}
\frac{\partial N_{\mathrm{Fe}}^{-}}{\partial t}= & -\left[q_{\mathrm{Fe}} s_{\mathrm{Fe}}+q_{\mathrm{Fe} X} s_{\mathrm{Fe} X}\left(N_{X}-N_{X}^{-}\right)\right] I_{G} N_{\mathrm{Fe}}^{-} \\
& +\left(\gamma_{\mathrm{Fe}} n+\gamma_{\mathrm{Fe} X} N_{X}^{-}\right)\left(N_{\mathrm{Fe}}-N_{\mathrm{Fe}}^{-}\right), \\
\frac{\partial N_{X}^{-}}{\partial t}= & -\left[\beta_{X}+q_{X, G} s_{X, G} I_{G}+q_{X, \mathrm{IR}} s_{X, \mathrm{IR}} I_{\mathrm{IR}}\right. \\
& \left.+\gamma_{X \mathrm{Fe}}\left(N_{\mathrm{Fe}}-N_{\mathrm{Fe}}^{-}\right)\right] N_{X}^{-} \\
& +\left(\gamma_{X} n+q_{\mathrm{Fe} X} s_{\mathrm{Fe} X} I_{G} N_{\mathrm{Fe}}^{-}\right)\left(N_{X}-N_{X}^{-}\right) .
\end{aligned}
$$

All symbols are introduced in Table I. Excitation of electrons from $\mathrm{Nb}_{\mathrm{Li}}^{4+}$ is possible by green light (wavelength $532 \mathrm{~nm}$ ) and by infrared light (wavelength $1064 \mathrm{~nm}$ ). Thus, we added in Eq. (4) a generation term to account for the presence of the infrared light. Some parameters have a subscript " $G$ " or "IR" to indicate whether they correspond to green or infrared light.

We treat the situation where the light intensity and therefore all other spatially dependent quantities vary only along one direction. The coordinate along this direction is $x$. Then, the current, continuity, charge, and Poisson equations are

$$
\begin{gathered}
j=e \mu n E+\kappa_{\mathrm{Fe}} N_{\mathrm{Fe}}^{-} I_{G}+\kappa_{X, G} N_{X}^{-} I_{G}+\kappa_{X, \mathrm{IR}} N_{X}^{-} I_{\mathrm{IR}}+ \\
\frac{\partial j}{\partial x}=-e\left(\frac{\partial N_{\mathrm{Fe}}^{-}}{\partial t}+\frac{\partial N_{X}^{-}}{\partial t}+\frac{\partial n}{\partial t}\right), \\
\rho=-e\left(N_{\mathrm{Fe}}^{-}+N_{X}^{-}+n-N_{A}\right), \\
\frac{\partial E}{\partial x}=\frac{\rho}{\epsilon \epsilon_{0}} .
\end{gathered}
$$

Drift, bulk photovoltaic, and diffusion currents are considered. All symbols are introduced in Table I.

Jermann and Otten determined a set of parameters, which describes excellently all photorefractive features of $\mathrm{LiNbO}_{3}: \mathrm{Fe}$ observed in the experiments with the green light at continuous-wave and at pulsed laser intensities [21]. Their parameter set will be also employed in this work. Thus, our model is immediately consistent with all usual photorefractive properties of $\mathrm{LiNbO}_{3}: \mathrm{Fe}$ for recording with light of one wavelength. Only two of the many parameters occurring in Eqs. (3)-(8) are new and unknown: $q_{X, \mathrm{IR}} s_{X, \mathrm{IR}}$ and $\kappa_{X, \mathrm{IR}}$, the photon absorption cross section and the bulk photovoltaic coefficient of $\mathrm{Nb}_{\mathrm{Li}}^{4+/ 5+}$ for excitations with infrared light. 
TABLE I. Units, meaning, and values of all quantities involved in the analysis of two-step holographic recording in $\mathrm{LiNbO}_{3}: \mathrm{Fe}$. Subscripts " 0 " and " 1 " are added in the text to the spatially dependent quantities to indicate zeroth and first Fourier components. Values in parentheses show standard values, which are valid if nothing else is mentioned.

\begin{tabular}{|c|c|c|c|}
\hline Quantity (unit) & Meaning & Value & Reference \\
\hline & Parameters of Fe & & \\
\hline$N_{\mathrm{Fe}}\left(\mathrm{m}^{-3}\right)$ & Total concentration of $\mathrm{Fe}$ & $1.2 \times 10^{25}$ & \\
\hline$N_{\mathrm{Fe}}^{-}\left(\mathrm{m}^{-3}\right)$ & Concentration of $\mathrm{Fe}^{2+}$ & variable & \\
\hline$q_{\mathrm{Fe}} s_{\mathrm{Fe}}\left(\mathrm{m}^{2} / \mathrm{J}\right)$ & $\begin{array}{l}\text { Absorption cross section of } \mathrm{Fe}^{2+} \text { for absorption of } \\
\text { a photon and excitation of an electron from } \mathrm{Fe}^{2+} \\
\text { into the conduction band (light wavelength } 532 \\
\mathrm{~nm} \text { ) }\end{array}$ & $1.0 \times 10^{-5}$ & {$[21]$} \\
\hline$\gamma_{\mathrm{Fe}}\left(\mathrm{m}^{3} / \mathrm{s}\right)$ & $\begin{array}{l}\text { Coefficient for recombination of conduction band } \\
\text { electrons with } \mathrm{Fe}^{2+}\end{array}$ & $1.65 \times 10^{-14}$ & {$[21]$} \\
\hline \multirow[t]{2}{*}{$-\kappa_{\mathrm{Fe}}\left(\mathrm{m}^{3} / \mathrm{V}\right)$} & $\begin{array}{l}\text { Bulk photovoltaic coefficient for excitation of electrons } \\
\text { from } \mathrm{Fe}^{2+} \text { into the conduction band (light } \\
\text { wavelength } 532 \mathrm{~nm} \text { ) }\end{array}$ & $3.5 \times 10^{-33}$ & {$[21]$} \\
\hline & \multicolumn{2}{|l|}{ Parameters of $\mathrm{Nb}_{\mathrm{Li}}$} & \multirow{3}{*}[19]{$,[20],[21]$} \\
\hline$N_{X}\left(\mathrm{~m}^{-3}\right)$ & Total concentration of $\mathrm{Nb}_{\mathrm{Li}}$ & $10^{26}$ & \\
\hline$N_{X}^{-}\left(\mathrm{m}^{-3}\right)$ & Concentration of $\mathrm{Nb}_{\mathrm{Li}}^{4+}$ & variable & \\
\hline$\beta_{X}\left(\mathrm{~s}^{-1}\right)$ & $\begin{array}{l}\text { Rate of thermal excitation of electrons from } \mathrm{Nb}_{\mathrm{Li}}^{4+} \\
\text { into the conduction band }\end{array}$ & 0 & {$[21]$} \\
\hline$q_{X, G} s_{X, G}\left(\mathrm{~m}^{2} / \mathrm{J}\right)$ & $\begin{array}{l}\text { Absorption cross section of } \mathrm{Nb}_{\mathrm{Li}}^{4+} \text { for absorption } \\
\text { of a photon and excitation of an electron into the } \\
\text { conduction band (light wavelength } 532 \mathrm{~nm} \text { ) }\end{array}$ & $5.0 \times 10^{-5}$ & {$[21]$} \\
\hline$q_{X, \mathrm{IR}} s_{X, \mathrm{IR}}\left(\mathrm{m}^{2} / \mathrm{J}\right)$ & $\begin{array}{l}\text { Absorption cross section of } \mathrm{Nb}_{\mathrm{Li}}^{4+} \text { for absorption } \\
\text { of a photon and excitation of an electron into the } \\
\text { conduction band (light wavelength } 1064 \mathrm{~nm} \text { ) }\end{array}$ & $5.4 \times 10^{-5}$ & [this work] \\
\hline$\gamma_{X}\left(\mathrm{~m}^{3} / \mathrm{s}\right)$ & $\begin{array}{l}\text { Coefficient for recombination of conduction band } \\
\text { electrons with } \mathrm{Nb}_{\mathrm{Li}}^{5+}\end{array}$ & 0 & {$[21]$} \\
\hline$-\kappa_{X, G}\left(\mathrm{~m}^{3} / \mathrm{V}\right)$ & $\begin{array}{l}\text { Bulk photovoltaic coefficient for excitation of electrons } \\
\text { from } \mathrm{Nb}_{\mathrm{Li}}^{4+} \text { into the conduction band (light } \\
\text { wavelength } 532 \mathrm{~nm} \text { ) }\end{array}$ & $21.2 \times 10^{-33}$ & {$[21]$} \\
\hline$-\kappa_{X, \mathrm{IR}}\left(\mathrm{m}^{3} / \mathrm{V}\right)$ & $\begin{array}{l}\text { Bulk photovoltaic coefficient for excitation of electrons } \\
\text { from } \mathrm{Nb}_{\mathrm{Li}}^{4+} \text { into the conduction band (light } \\
\text { wavelength } 1064 \mathrm{~nm} \text { ) }\end{array}$ & $32 \times 10^{-33}$ & [this work] \\
\hline$q_{\mathrm{FeX}} s_{\mathrm{FeX}}\left(\mathrm{m}^{5} / \mathrm{J}\right)$ & $\begin{array}{l}\text { Parameters related to } \mathrm{Fe} \text { and } \mathrm{Nb}_{\mathrm{Li}} \\
\text { Absorption cross section of } \mathrm{Fe}^{2+} \text { for absorption of } \\
\text { a photon and excitation of an electron into } \mathrm{Nb}_{\mathrm{Li}}^{4+} \\
\text { (light wavelength } 532 \mathrm{~nm} \text { ) }\end{array}$ & $3.22 \times 10^{-30}$ & {$[21]$} \\
\hline$\gamma_{X \mathrm{Fe}}\left(\mathrm{m}^{3} / \mathrm{s}\right)$ & $\begin{array}{l}\text { Coefficient for recombination of electrons from } \\
\mathrm{Nb}_{\mathrm{Li}}^{4+} \text { and } \mathrm{Fe}^{3+}\end{array}$ & $1.14 \times 10^{-21}$ & {$[21]$} \\
\hline & Parameters of $\mathrm{LiNbO}_{3}$ & & \\
\hline$\epsilon$ & Dielectric coefficient & 28 & {$[30],[31]$} \\
\hline$r_{13}(\mathrm{~m} / \mathrm{V})$ & $\begin{array}{l}\text { Electro-optic coefficient (light wavelength } 632.8 \\
\mathrm{~nm} \text { ) }\end{array}$ & $10.9 \times 10^{-12}$ & {$[32]$} \\
\hline$n_{0}$ & $\begin{array}{l}\text { Refractive index for ordinarily polarized light } \\
\text { (wavelength } 632 \mathrm{~nm} \text { ) }\end{array}$ & 2.286 & {$[33]$} \\
\hline & Charge transport parameters & & \\
\hline$j\left(\mathrm{~A} / \mathrm{m}^{2}\right)$ & Current density & variable & \\
\hline$\mu\left(\mathrm{m}^{2} / \mathrm{Vs}\right)$ & Electron mobility in the conduction band & $7.4 \times 10^{-5}$ & {$[34]$} \\
\hline$n\left(\mathrm{~m}^{-3}\right)$ & Density of free electrons in the conduction band & variable & \\
\hline$\rho\left(\mathrm{As} / \mathrm{m}^{3}\right)$ & Total charge density & variable & \\
\hline$N_{A}\left(\mathrm{~m}^{-3}\right)$ & $\begin{array}{l}\text { Concentration of nonmobile positive compensation } \\
\text { charge, which maintains overall charge neutrality }\end{array}$ & $\left(5.7 \times 10^{24}\right)$ & \\
\hline$E(\mathrm{~V} / \mathrm{m})$ & Space-charge field & variable & \\
\hline
\end{tabular}


TABLE I. (Continued).

\begin{tabular}{|c|c|c|c|}
\hline Quantity (unit) & Meaning & Value & Reference \\
\hline & Fundamental constants & & \\
\hline$k_{B}(\mathrm{~J} / \mathrm{K})$ & Boltzmann constant & $1.38 \times 10^{-23}$ & \\
\hline \multirow[t]{2}{*}{$\epsilon_{0}(\mathrm{As} / \mathrm{Vm})$} & Primitivity of free space & $8.85 \times 10^{-12}$ & \\
\hline & Parameters related to the experimental conditions & & \\
\hline$T(\mathrm{~K})$ & Crystal temperature & 293 & \\
\hline$K\left(\mathrm{~m}^{-1}\right)$ & Spatial frequency of the interference pattern & $2.9 \times 10^{6}$ & \\
\hline$\Lambda(\mathrm{m})$ & Period length of the interference pattern & $2.2 \times 10^{-6}$ & \\
\hline$I_{G}\left(\mathrm{~W} / \mathrm{m}^{2}\right)$ & $\begin{array}{l}\text { Intensity of the spatially homogeneous green light } \\
\text { (wavelength } 532 \mathrm{~nm} \text { ) }\end{array}$ & variable & \\
\hline$I_{\mathrm{IR}}\left(\mathrm{W} / \mathrm{m}^{2}\right)$ & Intensity of the infrared light (wavelength $1064 \mathrm{~nm}$ ) & variable & \\
\hline$m$ & $\begin{array}{l}\text { Modulation degree of the interference pattern of } \\
\text { the infrared light }\end{array}$ & variable & \\
\hline$t_{p}(s)$ & Duration of each green and infrared light pulse & $15 \times 10^{-9}$ & \\
\hline
\end{tabular}

To study this model, we will investigate the situation of simultaneous illumination with spatially homogeneous green light and with a sinusoidally modulated infrared interference pattern

$$
I_{\mathrm{IR}}=I_{\mathrm{IR}, 0}[1+m \sin (K x)] .
$$

The symbols are explained in Table I. We assume that the light intensity does not change with time during illumination. All calculations are performed with $m=0.1$ and the obtained space-charge fields are normalized to $m$, i.e., they are divided by $m$.

\section{NUMERICAL SOLUTION}

\section{A. Algorithm}

One may argue that typical approximations like the adiabatic approximation [27] or Fourier development with the neglect of higher Fourier orders [28] cannot be applied to our situation. Therefore, Eqs. (3)-(9) are solved numerically in space without any approximation. The calculations are performed for one period length of the grating, and cyclic boundary conditions are used.

The starting condition is the steady-state situation in the dark with a homogeneous concentration of $\mathrm{Fe}^{2+}$, which is equal to the concentration of compensators (or acceptors) $N_{A}$, because the $\mathrm{Nb}_{\mathrm{Li}}$ centers are initially not populated, i.e., $N_{X}^{-}=0$. Calculations are done in time steps $d t$ : First, the concentration patterns $N_{\mathrm{Fe}}^{-}(x, t+d t)$ and $N_{X}^{-}(x, t+d t)$ are calculated using Eqs. (3) and (4), and the values $N_{\mathrm{Fe}}^{-}(x, t)$ and $N_{X}^{-}(x, t)$. The current density $j(x, t)$ is calculated from Eq. (5) and the concentration pattern $n(x, t+d t)$ is finally obtained from $n(x, t)$ and from Eqs. (3), (4), and (6). Then, Eq. (7) and the integration of Eq. (8) finally yield the spacecharge field $E(x, t+d t)$. This cycle is periodically repeated until the end of one light pulse is reached. The typical repetition frequency of the pulsed lasers used in the experiments is low, i.e., around $10 \mathrm{~Hz}$. The time between the pulses is sufficient that all electrons that were excited to $\mathrm{Nb}_{\mathrm{Li}}^{4+}$ recom- bine locally with $\mathrm{Fe}^{3+}$. Thus, the program adds to $N_{\mathrm{Fe}}^{-}$the actual $N_{X}^{-}$values at the end of each pulse and sets $N_{X}^{-}$to zero afterwards. The created refractive index changes for ordinarily polarized red light (wavelength $632.8 \mathrm{~nm}$ ) are calculated with $\Delta n(x)=-(1 / 2) n_{0}^{3} r_{13} E(x)$, using the parameters introduced in Table I.

The time steps are always chosen so small that further reduction has no influence on the calculated results. A typical time step for the calculations is $1 \mathrm{ps}$, and 100 points in space are used to represent one period length of the interference pattern.

Numerical solution of the high intensity properties, as is done here, benefits from one fact: the concentration of the electrons in the conduction band $n$ is two or three orders of magnitude smaller than the defect concentrations. The difference is much larger for low light intensities, and $n$ cannot be obtained in the way described above because of limited calculation accuracy, i.e., $n$ is the tiny difference of two large and almost completely compensating rates. Anyhow, the algorithm is fine for pulsed illumination and no approximations have to be introduced.

\section{B. Shape and evolution of the space-charge field}

Figure 4 shows the space-charge field pattern for different times. The space-charge field is a replica of the light pattern and has an almost perfect sinusoidal shape because of the low modulation depth $(m=0.1)$ used in these simulations. Thus, the amplitude of the space-charge field modulation can be easily determined from a sinusoidal fit to the computed data. This result is a first indication that Fourier development will be a useful approach for obtaining an analytical solution to the problem.

The evolution of the space-charge field amplitude during recording and erasure is presented in Fig. 5. No electrons are in the $\mathrm{Nb}_{\mathrm{Li}}^{4+15+}$ centers at the beginning of each light pulse. Thus, the green light starts to erase the previously written hologram due to direct excitation of electrons into the conduction band and the created conductivity. However, the 


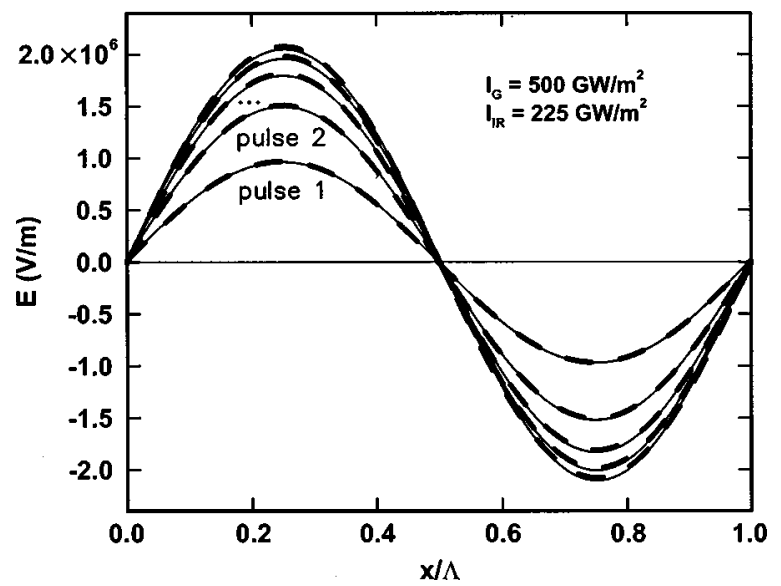

FIG. 4. Space charge field $E$ versus spatial coordinate $x$ normalized to the grating period length $\Lambda$. The solid line shows the computed space-charge field after illumination with one, two, three, four, and five light pulses of high intensity $\left(I_{G}=500 \mathrm{GW} / \mathrm{m}^{2}, I_{\mathrm{IR}}\right.$ $=225 \mathrm{GW} / \mathrm{m}^{2}$ ). The dashed lines are sinusoidal fits to the calculated curves.

$\mathrm{Nb}_{\mathrm{Li}}^{4+/ 5+}$ is populated more and more during the pulse. The infrared light excites electrons from this level into the conduction band, a modulated bulk photovoltaic current arises, and the space-charge field grows. These processes are the origin of what we observe in the saturation regime, i.e., after long recording times, during each pulse at first a decrease and then an increase of the space-charge field amplitude. Saturation means that erasure and recording effects compensate each other completely. From Fig. 5 it becomes also clear that the evolution of the space-charge field during the pulse illumination can be very well approximated by a parabolic function. Furthermore, it can be seen that considering the fields at the end of each pulse, growth and erasure of the grating are described by monoexponential functions.

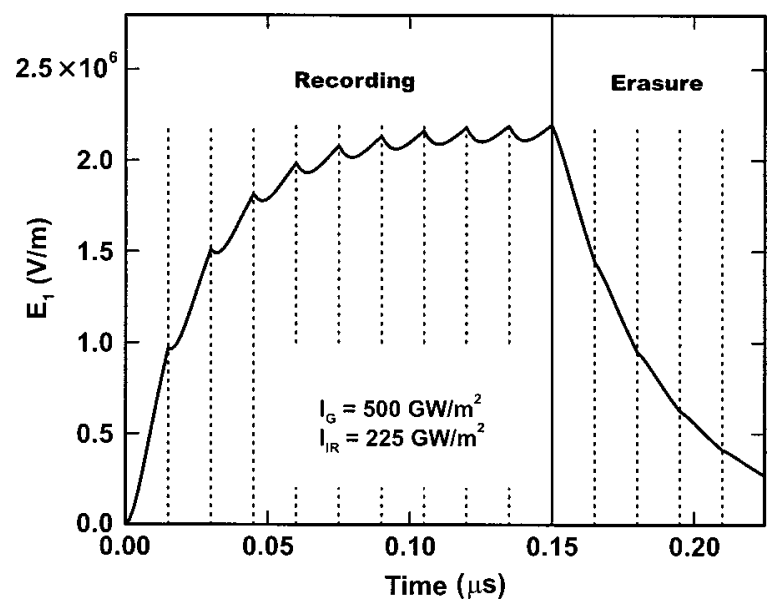

FIG. 5. Amplitude $E_{1}$ of the space-charge field versus exposure time for recording and erasure. The light intensities are $I_{G}$ $=500 \mathrm{GW} / \mathrm{m}^{2}$ and $I_{\mathrm{IR} 0}=225 \mathrm{GW} / \mathrm{m}^{2}$. The averaged light intensities are equal for recording and erasure. The thin vertical lines indicate the end of each 15-ns-long pulse.

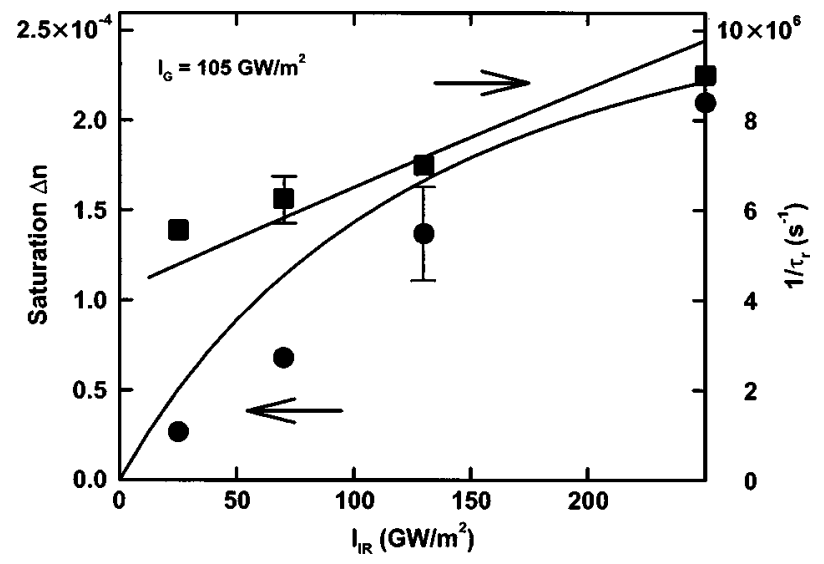

FIG. 6. Variation of the saturation value of the amplitude of the refractive index grating $(\Delta n)$ and recording speed $\tau_{r}^{-1}$ (inverse of recording time constant) with average infrared light intensity $I_{\text {IR }}$ (with constant $I_{G}=105 \mathrm{GW} / \mathrm{m}^{2}$ ). The curves are calculated in terms of the two-center model and the symbols are experimental data.

\section{Intensity and concentration dependences}

The dependence of the saturation value of the created refractive index modulations and of the recording time constant on the intensities of green and infrared light were experimentally investigated $[10,12]$. Furthermore, the influence of the initial homogeneous $\mathrm{Fe}^{2+}$ concentration on the sensitivity, i.e., on the change of the refractive index amplitude per unit time at the beginning of the recording, and of the initial homogeneous concentration of $\mathrm{Fe}^{3+}$ on the saturation values of the refractive index changes were also carefully determined in several experiments $[10,12]$.

Only two parameters remain free and can be varied in order to explain all these dependences, the photon absorption cross section $q_{X \text {.IR }} s_{X, \mathrm{IR}}$ and the bulk photovoltaic coefficient $\kappa_{X, \mathrm{IR}}$ of the $\mathrm{Nb}_{\mathrm{Li}}^{4+15+}$ center for infrared light. Figures 6-9 show impressively that all experimental results mentioned above can be excellently described by proper selection of

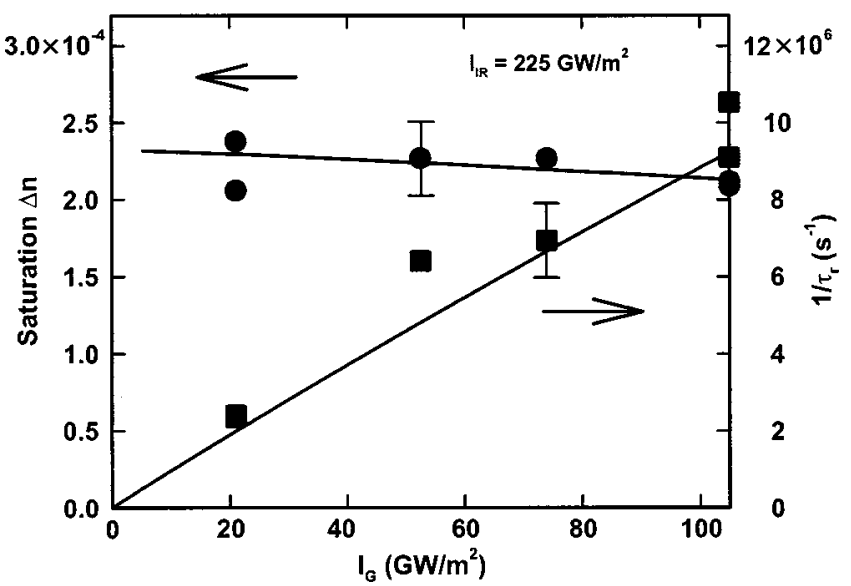

FIG. 7. Variation of the saturation value of the amplitude of the refractive index grating $(\Delta n)$ and recording speed $\tau_{r}^{-1}$ (inverse of recording time constants) with green light intensity $I_{G}$ (with constant $I_{\mathrm{IR}}=225 \mathrm{GW} / \mathrm{m}^{2}$ ). The curves are calculated in terms of the two-center model and the symbols are experimental data. 


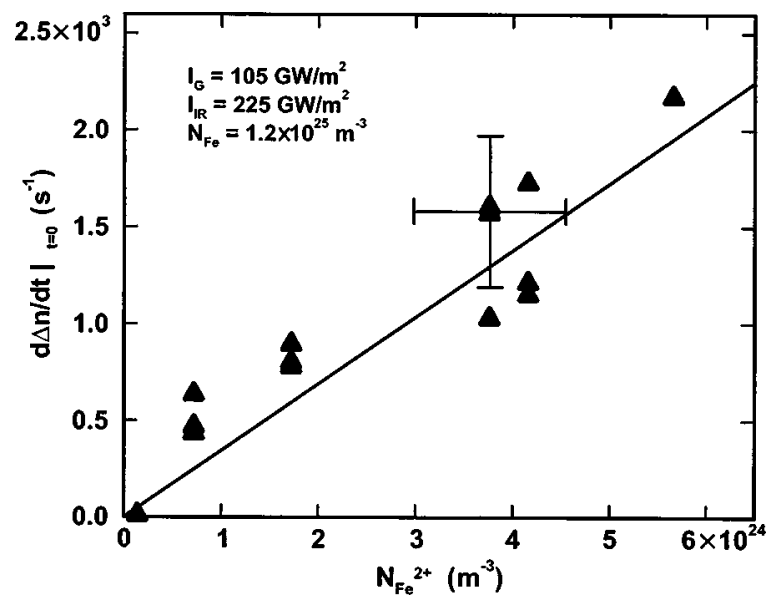

FIG. 8. Variation of sensitivity (changes of the amplitude of the refractive index changes per time at the beginning of recording, $d \Delta n /\left.d t\right|_{t=0}$ ) with the averaged concentration of $\mathrm{Fe}^{2+}, N_{\mathrm{Fe}^{2+}}$ (that is equal to $N_{A}$ ). The light intensities are $I_{G}=105 \mathrm{GW} / \mathrm{m}^{2}$ and $I_{\mathrm{IR}}$ $=225 \mathrm{GW} / \mathrm{m}^{2}$. The curve is calculated in terms of the two-center model and the symbols are experimental data.

just these two parameters. To find these two parameters, we varied them over a wide range to obtain good agreement between the theoretical and the experimental results as shown in Figs. 6-9. The results obtained for $q_{X, \mathrm{IR}} s_{X, \mathrm{IR}}$ and $\kappa_{X, \mathrm{IR}}$ are shown in Table I. This success is a clear indication that the model is appropriate and that the determined parameters are very reliable. In the next sections, we will develop an analytic solution and will use it to explain the experimental results.

\section{ANALYTIC SOLUTION}

In this section, we develop an approximate analytic solution for Eqs. (3)-(8). To do this, we need some assumptions

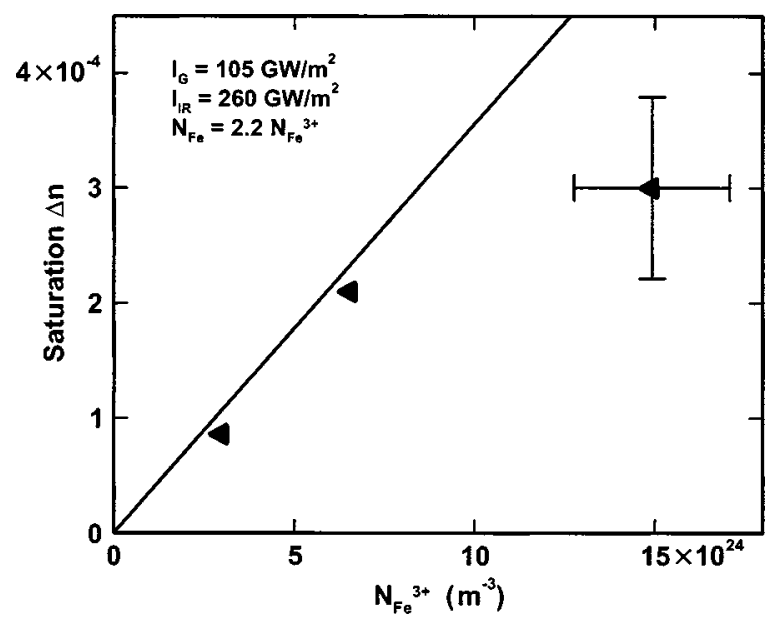

FIG. 9. Variation of the saturation value of the amplitude of the refractive index grating $(\Delta n)$ with concentration of $\mathrm{Fe}^{3+}, N_{\mathrm{Fe}^{3+}}$ (that is equal to $N_{\mathrm{Fe}}-N_{A}$ ). It is assumed that the iron concentration increases according to $N_{\mathrm{Fe}}=2.2 \times N_{\mathrm{Fe}^{3+}}$. The light intensities are $I_{G}=105 \mathrm{GW} / \mathrm{m}^{2}$ and $I_{\mathrm{IR}}=260 \mathrm{GW} / \mathrm{m}^{2}$. The curve is calculated in terms of the two-center model and the symbols are experimental data. to simplify the equations. We can test the validity of each assumption by comparing results of the complete numerical solutions with and without that assumption.

\section{A. Assumptions}

Assumption 1: We neglect the trapping of the conductionband electrons by the shallow traps during one pulse width. Therefore, we assume that the shallow traps are mainly populated by direct electron transfer from the deep traps, and the conduction-band electrons are mainly trapped by the deep traps.

Assumption 2: We neglect thermal depopulation of the shallow traps within one pulse at room temperature. This is a valid assumption, as the lifetime of the electrons in shallow traps is normally a few milliseconds, while the pulse width is typically a few nanoseconds.

Assumption 3: We neglect direct electron transfer (recombination) from shallow traps to deep traps within one pulse width. This is a valid assumption due to the same reason as in assumption 2. Combining assumptions 2 and 3 is equivalent to assuming that the depopulation of the shallow traps within one pulse width (a few nanoseconds) is negligible.

Assumption 4: We assume that any change in the concentration of electrons in the conduction band gets to steady state much faster than that in the concentration of electrons in either trap. Therefore, in the time scale of the variation of electrons in the traps, we can assume $\partial n / \partial t=0$. This is called the adiabatic approximation [27]. Numerical solutions of the system of differential equations with and without this assumption are practically the same. This fact has been reported by other authors, too (Ref. [21]).

Assumption 5: We assume that the electron concentration in the conduction band $(n)$ is much smaller than that in the deep and shallow traps $\left(N_{\mathrm{Fe}}^{-}\right.$and $N_{X}^{-}$, respectively) as well as $\left(N_{\mathrm{Fe}}^{-}+N_{X}^{-}-N_{A}\right)$. So, we neglect $n$ in Eq. (7).

Assumption 6: We neglect the diffusion term in Eq. (5). This is a valid assumption in $\mathrm{LiNbO}_{3}$, since the major source of the current is bulk photovoltaic current in the transmission geometry.

Numerical solutions of the governing differential equations are practically the same with and without these assumptions. In the next section, we add more approximations to get an analytic solution set for Eqs. (3)-(8).

Assumption 7: We assume that the sample is shortcircuited, i.e., the electric field $(E)$ does not have any dc component.

\section{B. Fourier development}

We assume that with sinusoidal intensity variation [Eq. (9)] each variable in Eqs. (3)-(8) can be represented by the first two terms in its Fourier series expansion. For example, the concentration of electrons in the deep traps $\left(N_{\mathrm{Fe}}^{-}\right)$can be represented as

$$
N_{\mathrm{Fe}}^{-}=N_{\mathrm{Fe} 0}^{-}+N_{\mathrm{Fe} 1}^{-} \exp (i K x) .
$$

Using this assumption, we can replace $\partial / \partial x$ by zero for the zero-order variables (e.g., $N_{\mathrm{Fe} 0}^{-}$) and by $i K$ for the first-order 
ones (e.g., $\left.N_{\mathrm{Fe} 1}^{-}\right)$. Replacing every variable in Eqs. (3)-(8) with its first two Fourier expansion terms and separating the equations for the zero- and first-order variables, we obtain the following two sets of equations:

$$
\begin{aligned}
& \frac{d N_{\mathrm{Fe} 0}^{-}}{d t}=-\left[q_{\mathrm{Fe}} s_{\mathrm{Fe}}+q_{\mathrm{Fe} X} s_{\mathrm{Fe} X}\left(N_{X}-N_{X 0}^{-}\right)\right] I_{G} N_{\mathrm{Fe} 0}^{-} \\
&+\gamma_{\mathrm{Fe}} n_{0}\left(N_{\mathrm{Fe}}-N_{\mathrm{Fe} 0}^{-}\right) \\
& \frac{d N_{X 0}^{-}}{d t}=-\left(q_{X, G} s_{X, G} I_{G}+q_{X, \mathrm{IR}} s_{X, \mathrm{IR}} I_{\mathrm{IR} 0}\right) N_{X 0}^{-} \\
&+q_{\mathrm{Fe} X} s_{\mathrm{Fe} X} I_{G} N_{\mathrm{Fe} 0}^{-}\left(N_{X}-N_{X 0}^{-}\right) \\
& \frac{d N_{\mathrm{Fe} 0}^{-}}{d t}+\frac{d N_{X 0}^{-}}{d t}=0 \\
& N_{\mathrm{Fe} 0}^{-}+N_{X 0}^{-}=N_{A}
\end{aligned}
$$

for the zero-order variables, and

$$
\begin{aligned}
\frac{d N_{\mathrm{Fe} 1}^{-}}{d t}= & -\left(\left[q_{\mathrm{Fe}} s_{\mathrm{Fe}}+q_{\mathrm{Fe} X} s_{\mathrm{Fe} X}\left(N_{X}-N_{X 0}^{-}\right)\right] I_{G}+\gamma_{\mathrm{Fe}} n_{0}\right) N_{\mathrm{Fe} 1}^{-} \\
& +\gamma_{\mathrm{Fe}} n_{1}\left(N_{\mathrm{Fe}}-N_{\mathrm{Fe} 0}^{-}\right)+q_{\mathrm{Fe} X} s_{\mathrm{Fe} X} N_{\mathrm{Fe} 0}^{-} I_{G} N_{X 1}^{-}
\end{aligned}
$$

$$
\begin{aligned}
& \frac{d N_{X 1}^{-}}{d t}=-\left(q_{X, G} s_{X, G} I_{G}+q_{X, \mathrm{IR}} s_{X, \mathrm{IR}} I_{\mathrm{IR} 0}\right. \\
&\left.+q_{\mathrm{Fe} X} s_{\mathrm{Fe} X} I_{G} N_{\mathrm{Fe} 0}^{-}\right) N_{X 1}^{-}+q_{\mathrm{Fe} X} s_{\mathrm{Fe} X} I_{G}\left(N_{X}-N_{X 0}^{-}\right) N_{\mathrm{Fe} 1}^{-} \\
&-q_{X, \mathrm{IR}} s_{X, \mathrm{IR}} N_{X 0}^{-} I_{\mathrm{IR} 1}, \\
& j_{1}=\frac{i e}{K}\left(\frac{d N_{\mathrm{Fe} 1}^{-}}{d t}+\frac{d N_{X 1}^{-}}{d t}\right), \\
& j_{1}= e \mu n_{0} E_{1}+\kappa_{\mathrm{Fe}} I_{G} N_{\mathrm{Fe} 1}^{-}+\left(\kappa_{X, G} I_{G}+\kappa_{X, \mathrm{IR}} I_{\mathrm{IR} 0}\right) N_{X 1}^{-} \\
&+\kappa_{X, \mathrm{IR}} N_{X 0}^{-} I_{\mathrm{IR} 1}, \\
& E_{1}= \frac{-i e}{K \epsilon \epsilon_{0}}\left(N_{\mathrm{Fe} 1}^{-}+N_{X 1}^{-}\right),
\end{aligned}
$$

for the first-order variables. The goal is to find the first Fourier term of the space-charge field $\left(E_{1}\right)$ that can be used to find the change in the index of refraction through electrooptic effect. To find $E_{1}$, we first need to solve the equations for the zero-order variables [Eqs. (11)-(14)]. We can then put the zero-order variables into the first-order equations and find $E_{1}$. To check the validity of the above assumptions, we solved the given zero- and first-order equations (with all assumptions applied) numerically. Figure 10 shows the variation of the space-charge field $E_{1}$ with time during recording. The same variation calculated by the exact numerical solution is also shown in Fig. 10, confirming the validity of all assumptions and approximations.

Note that the use of only two Fourier components (zero and first order) for each variable in the governing equations

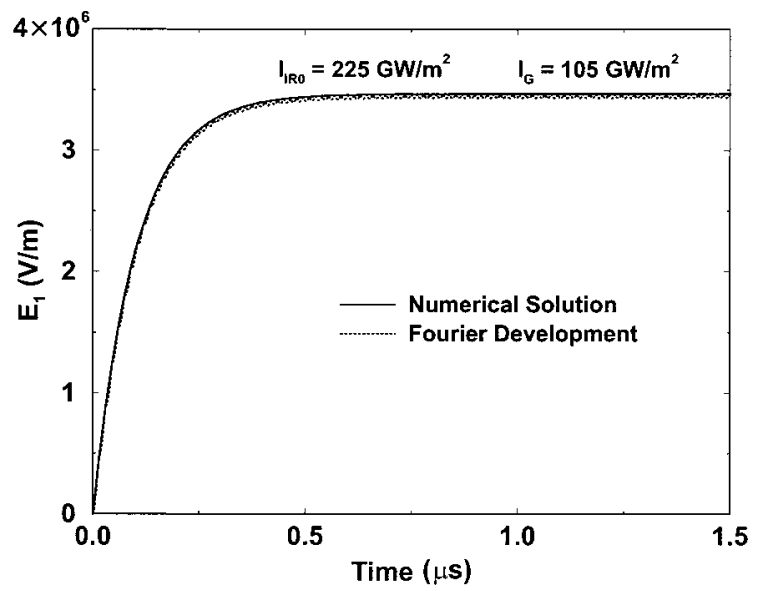

FIG. 10. Theoretical calculation of the space-charge field versus time during recording of a hologram using two-step recording. The two curves are calculated using the complete numerical solution and the approximate solution based on Fourier development with several assumptions given in the text. The agreement between the curves is excellent. The light intensities used in these calculations are $I_{G}=105 \mathrm{GW} / \mathrm{m}^{2}$ and $I_{\mathrm{IR} 0}=I_{\mathrm{IR} 1}=225 \mathrm{GW} / \mathrm{m}^{2}$.

is valid only for small intensity modulation depth (up to $m$ $=0.8$ ) [29]. For larger modulation depths, the space-charge field calculated by using the Fourier development (with only two terms for each variable) is smaller than the actual value (calculated by full numerical solution) by as much as $30 \%$ (at $m=1$ ). On the other hand, modulation depths larger than $m=0.8$ are hard to achieve experimentally (even with equal intensity beams) due to the multiple reflections of the recording beams at the entrance and exit faces of the crystal. These reflections reduce the modulation depth by increasing the dc light intensity. Therefore, the actual experimental modulation depth for equal intensity beams is about $m \simeq 0.8$, and the actual experimental space-charge field is smaller than that calculated using $m=1$ by about $20 \%$. This makes the Fourier development (with the first two Fourier terms of each variable) a better approximation than the complete numerical solution for the actual measured values of space-charge field at high modulation depths. A similar argument holds for the validity of assuming linear variation of the space-charge field with the modulation depth $m$ that we used in the numerical solution.

\section{Solution of the zero-order equations}

To solve the zero-order equations, we first put Eqs. (11) and (12) into Eq (13) and use $N_{\mathrm{Fe} 0}^{-}=N_{A}-N_{X 0}^{-}$from Eq. (14) to find $n_{0}$ in terms of $N_{X 0}^{-}$,

$$
=\frac{q_{\mathrm{Fe}} s_{\mathrm{Fe}} I_{G} N_{A}+\left(q_{X, G} s_{X, G} I_{G}+q_{X, \mathrm{IR}} s_{X, \mathrm{IR}} I_{\mathrm{IR} 0}-q_{\mathrm{Fe}} s_{\mathrm{Fe}} I_{G}\right) N_{X 0}^{-}}{\gamma_{\mathrm{Fe}}\left(N_{\mathrm{Fe}}-N_{A}+N_{X 0}^{-}\right)} .
$$

Therefore, we only need to solve for $N_{X 0}^{-}$. This can be done by putting $N_{\mathrm{Fe} 0}^{-}=N_{A}-N_{X 0}^{-}$into Eq. (12) to obtain 


$$
\begin{aligned}
\frac{d N_{X 0}^{-}}{d t}= & q_{\mathrm{Fe} X} s_{\mathrm{Fe} X} I_{G}\left(N_{X 0}^{-}\right)^{2} \\
& -\left[\left(q_{X, G} s_{X, G}+q_{\mathrm{Fe} X} s_{\mathrm{Fe} X}\left(N_{X}+N_{A}\right)\right) I_{G}\right. \\
& \left.+q_{X, \mathrm{IR}} s_{X, \mathrm{IR}} I_{\mathrm{IR} 0}\right] N_{X 0}^{-}+q_{\mathrm{Fe} X} s_{\mathrm{Fe} X} I_{G} N_{X} N_{A},
\end{aligned}
$$

with the initial condition being $N_{X 0}^{-}(t=0)=0$.

Assumption 8: We assume that

$$
q_{\mathrm{Fe} X} s_{\mathrm{Fe} X} I_{G}\left(N_{X 0}^{-}\right)^{2} \ll q_{\mathrm{Fe} X} s_{\mathrm{Fe} X}\left(N_{X}+N_{A}\right) N_{X 0}^{-}
$$

or

$$
N_{X 0}^{-} \ll N_{X}+N_{A}
$$

Since we usually have $N_{A} \ll N_{X}$, the assumption of Eq. (23) is equivalent to assuming that only a very small portion of the shallow traps is populated during one pulse width by electron transfer from the deep traps. Using this assumption, we can neglect $q_{\mathrm{FeX}} s_{\mathrm{Fe} X} I_{G}\left(N_{X 0}^{-}\right)^{2}$ in Eq. (21) and solve for $N_{X 0}^{-}$to obtain

$$
N_{X 0}^{-}=\frac{q_{\mathrm{Fe} X} s_{\mathrm{Fe} X} I_{G} N_{X} N_{A}\left[1-\exp \left(-t / \tau_{x}\right)\right]}{q_{X, G} s_{X, G} I_{G}+q_{X, \mathrm{IR}} s_{X, \mathrm{IR}} I_{\mathrm{IR} 0}+q_{\mathrm{Fe} X} s_{\mathrm{Fe} X}\left(N_{X}+N_{A}\right) I_{G}},
$$

where

$$
\tau_{x}=\frac{1}{q_{X, G} s_{X, G} I_{G}+q_{X, \mathrm{IR}} s_{X, \mathrm{IR}} I_{\mathrm{IR} 0}+q_{\mathrm{Fe} X} s_{\mathrm{Fe} X}\left(N_{X}+N_{A}\right) I_{G}} .
$$

Using the parameter values from Table I and assuming sensitizing and recording intensities $\left(I_{G}\right.$ and $\left.I_{\mathrm{IR} 0}\right)$ of about $10^{12} \mathrm{~W} / \mathrm{m}^{2}$, we obtain $\tau_{x} \simeq 100 \mathrm{~ns}$. For a pulse width of $t_{p}$ $\simeq 15 \mathrm{~ns}$, we can calculate $1-\exp \left(-t_{p} / \tau_{x}\right) \simeq 0.14$. For $I_{G}$ $\simeq I_{\mathrm{IR} 0} \simeq 10^{11} \mathrm{~W} / \mathrm{m}^{2}$, and the same pulse width, we obtain 1 $-\exp \left(-t_{p} / \tau_{x}\right) \simeq 0.015$. Therefore, we can use the following approximation for the time within one pulse width $\left(t \leqslant t_{p}\right)$ :

$$
1-\exp \left(-t / \tau_{x}\right) \simeq \frac{t}{\tau_{x}}
$$

With this approximation, we can summarize the zero-order variables as

$$
\begin{gathered}
N_{X 0}^{-}=q_{\mathrm{Fe} X} s_{\mathrm{Fe} X} I_{G} N_{X} N_{A} t, \\
N_{\mathrm{Fe} 0}^{-}=N_{A}-q_{\mathrm{Fe} X} s_{\mathrm{Fe} X} I_{G} N_{X} N_{A} t
\end{gathered}
$$

$$
\begin{aligned}
n_{0} & =\frac{q_{\mathrm{Fe}} s_{\mathrm{Fe}} I_{G} N_{A}+\left(q_{X, G} s_{X, G} I_{G}+q_{X, \mathrm{IR}} s_{X, \mathrm{IR}} I_{\mathrm{IR} 0}-q_{\mathrm{Fe}} s_{\mathrm{Fe}} I_{G}\right) N_{X 0}^{-}}{\gamma_{\mathrm{Fe}}\left(N_{\mathrm{Fe}}-N_{A}+N_{X 0}^{-}\right)} \\
& =\frac{q_{\mathrm{Fe}} s_{\mathrm{Fe}} I_{G} N_{A}}{\gamma_{\mathrm{Fe}}\left(N_{\mathrm{Fe}}-N_{A}\right)}+\frac{q_{X, G} s_{X, G} I_{G}+q_{X, \mathrm{IR}} s_{X, \mathrm{IR}} I_{\mathrm{IR} 0}-\frac{N_{\mathrm{Fe}}}{N_{\mathrm{Fe}}-N_{A}} q_{\mathrm{Fe}} s_{\mathrm{Fe}} I_{G}}{\gamma_{\mathrm{Fe}}\left(N_{\mathrm{Fe}}-N_{A}\right)} q_{\mathrm{Fe} X} s_{\mathrm{Fe} X} I_{G} N_{X} N_{A} t=n_{00}+n_{01} t,
\end{aligned}
$$

\section{Solution of the first-order equations}

We can put the solutions of the zero-order equations into first-order equations [Eqs. (15)-(19)] and solve them. To solve the first-order equations, we first combine Eqs. (18) and (19) to obtain

$$
\begin{aligned}
j_{1}= & -\frac{i e^{2} \mu n_{0}}{K \epsilon \epsilon_{0}}\left(N_{\mathrm{Fe} 1}^{-}+N_{X 1}^{-}\right)+\kappa_{\mathrm{Fe}} I_{G} N_{\mathrm{Fe} 1}^{-}+\left(\kappa_{X, G} I_{G}\right. \\
& \left.+\kappa_{X, \mathrm{IR}} I_{\mathrm{IR} 0}\right) N_{X 1}^{-}+\kappa_{X, \mathrm{IR}} N_{X 0}^{-} I_{\mathrm{IR} 1} .
\end{aligned}
$$

terms in its Taylor series expansion around $t=0$. In other words, we could have approximated each variable during one pulse width by a simple linear function of time (i.e., $C_{1}$ $\left.+C_{2} t\right)$. The solution of the zero-order equations would then consist of finding the unknown constants (i.e., $C_{1}$ and $C_{2}$ ).
Furthermore, any term that included $\left(N_{X 0}^{-}\right)^{2}$ was neglected. suming that the variables do not change much during one pulse width and approximating each variable by the first two
Then, we put Eqs. (15), (16), and (31) into Eq. (17), and solve for $n_{1}$ as a function of $N_{\mathrm{Fe} 1}^{-}$and $N_{X 1}^{-}$. The result is 


$$
\begin{aligned}
n_{1}=- & \frac{\frac{e \mu n_{0}}{\epsilon \epsilon_{0}}+q_{X, G} s_{X, G} I_{G}+q_{X, \mathrm{IR}} s_{X, \mathrm{IR}} I_{\mathrm{IR} 0}-\left(\frac{i K}{e}\right)\left(\kappa_{X, G} I_{G}+\kappa_{X, \mathrm{IR}} I_{\mathrm{IR} 0}\right)}{\gamma_{\mathrm{Fe}}\left(N_{\mathrm{Fe}}-N_{A}+N_{X 0}^{-}\right)} N_{X 1}^{-} \\
& +\frac{-\frac{e \mu n_{0}}{\epsilon \epsilon_{0}}+q_{\mathrm{Fe}} s_{\mathrm{Fe}} I_{G}+\gamma_{\mathrm{Fe}} n_{0}-\frac{i K}{e} \kappa_{\mathrm{Fe}} I_{G}}{\gamma_{\mathrm{Fe}}\left(N_{\mathrm{Fe}}-N_{A}+N_{X 0}^{-}\right)} N_{\mathrm{Fe} 1}^{-}+\frac{\left(q_{X, \mathrm{IR}} s_{X, \mathrm{IR}}-\frac{i K}{e} \kappa_{X, \mathrm{IR}}\right) N_{X 0}^{-} I_{\mathrm{IR} 1}}{\gamma_{\mathrm{Fe}}\left(N_{\mathrm{Fe}}-N_{A}+N_{X 0}^{-}\right)} .
\end{aligned}
$$

The next step is to substitute $n_{1}$ from Eq. (32) into Eqs. (15) and (16), and combine these two equations to obtain a set of two ordinary differential equations for two unknowns $N_{X 1}^{-}$ and $N_{\mathrm{Fe} 1}^{-}+N_{X 1}^{-}$as

$$
\begin{aligned}
\frac{d\left(N_{\mathrm{Fe} 1}^{-}+N_{X 1}^{-}\right)}{d t}= & -\left[\frac{e \mu\left(n_{00}+n_{01} t\right)}{\epsilon \epsilon_{0}}+\frac{i K}{e} \kappa_{\mathrm{Fe}} I_{G}\right]\left(N_{\mathrm{Fe} 1}^{-}+N_{X 1}^{-}\right) \\
& -\frac{i K}{e}\left(\kappa_{X, G} I_{G}+\kappa_{X, \mathrm{IR}} I_{\mathrm{IR} 0}-\kappa_{\mathrm{Fe}} I_{G}\right) N_{X 1}^{-} \\
& -\frac{i K}{e} \kappa_{X, \mathrm{IR}} I_{\mathrm{IR} 1}\left(q_{\mathrm{Fe} X} s_{\mathrm{Fe} X} I_{G} N_{X} N_{A}\right) t
\end{aligned}
$$

$$
\begin{aligned}
\frac{d N_{X 1}^{-}}{d t}= & q_{\mathrm{Fe} X} s_{\mathrm{Fe} X} I_{G}\left[N_{X}-\left(q_{\mathrm{Fe} X} s_{\mathrm{Fe} X} I_{G} N_{X} N_{A}\right) t\right]\left(N_{\mathrm{Fe} 1}^{-}+N_{X 1}^{-}\right) \\
& -\left(q_{X, G} s_{X, G} I_{G}+q_{X, \mathrm{IR}} s_{X, \mathrm{IR}} I_{\mathrm{IR} 0}\right) N_{X 1}^{-} \\
& +q_{\mathrm{Fe} X} s_{\mathrm{Fe} X} I_{G}\left[N_{X}+N_{A}-2\left(q_{\mathrm{Fe} X} s_{\mathrm{Fe} X} I_{G} N_{X} N_{A}\right) t\right] N_{X 1}^{-} \\
& -q_{X, \mathrm{IR}} s_{X, \mathrm{IR}} I_{\mathrm{IR} 1}\left(q_{\mathrm{Fe} X} s_{\mathrm{Fe} X} I_{G} N_{X} N_{A}\right) t
\end{aligned}
$$

where we replaced the zero-order variables $\left(N_{X 0}^{-}, N_{\mathrm{Fe} 0}^{-}\right.$, and $n_{0}$ ) by their equivalents from Eqs. (27)-(29). Note that we deliberately chose $N_{\mathrm{Fe} 1}^{-}+N_{X 1}^{-}$as one variable since it is related to the space-charge field as

$$
E_{1}=-\frac{i e}{K \epsilon \epsilon_{0}}\left(N_{\mathrm{Fe} 1}^{-}+N_{X 1}^{-}\right) .
$$

The initial conditions for Eqs. (33) and (34) are

$$
\begin{aligned}
& N_{\mathrm{Fe} 1}^{-}(t=0)=A, \\
& N_{X 1}^{-}(t=0)=0,
\end{aligned}
$$

where we assumed that all electrons in the shallow traps are transferred to the deep traps in the time interval between adjacent pulses resulting in fully empty shallow traps at the beginning of every pulse $(t=0)$. The value of $N_{\mathrm{Fe} 1}^{-}$at the beginning of each pulse $(A)$ depends on time (or the total number of previous pulses) as space-charge is built up in $\mathrm{Fe}$ traps with time.

Assumption 9: We assume that the variations in first-order variables (i.e., $N_{\mathrm{Fe} 1}^{-}$and $N_{X 1}^{-}$) within one pulse width are small. Therefore, we can approximate every first-order variable with the first few terms in its Taylor-series expansion about $t=0$. Since the right-hand sides of Eqs. (33) and (34) contain terms like $C_{1}+C_{2} t$, we approximate both $N_{\mathrm{Fe} 1}^{-}$ $+N_{X 1}^{-}$and $N_{X 1}^{-}$by the first three terms in their Taylor-series expansions. Using the initial conditions given by Eqs. (36) and (37) and assuming that the pulse starts at time $t=0$, we can write

$$
\begin{gathered}
N_{\mathrm{Fe} 1}^{-}+N_{X 1}^{-}=A+B t+C t^{2}, \\
N_{X 1}^{-}=D t+E t^{2} .
\end{gathered}
$$

Putting Eqs. (38)-(39) into Eqs. (33)-(34), we obtain

$$
\begin{aligned}
B+2 C t= & -\left(\frac{e \mu\left(n_{00}+n_{01} t\right)}{\epsilon \epsilon_{0}}+\frac{i K}{e} \kappa_{\mathrm{Fe}} I_{G}\right)\left(A+B t+C t^{2}\right) \\
& -\frac{i K}{e}\left(\kappa_{X, G} I_{G}+\kappa_{X, \mathrm{IR}} I_{\mathrm{IR} 0}-\kappa_{\mathrm{Fe}} I_{G}\right)\left(D t+E t^{2}\right) \\
& -\frac{i K}{e} \kappa_{X, \mathrm{IR}} I_{\mathrm{IR} 1}\left(q_{\mathrm{Fe} X} s_{\mathrm{Fe} X} I_{G} N_{X} N_{A}\right) t
\end{aligned}
$$

$$
\begin{aligned}
D+2 E t= & q_{\mathrm{Fe} X} s_{\mathrm{Fe} X} I_{G}\left[N_{X}-\left(q_{\mathrm{Fe} X} s_{\mathrm{Fe} X} I_{G} N_{X} N_{A}\right) t\right] \\
& \times\left(A+B t+C t^{2}\right)-\left(q_{X, G} s_{X, G} I_{G}+q_{X, \mathrm{IR}} s_{X, \mathrm{IR}} I_{\mathrm{IR} 0}\right) \\
& \times\left(D t+E t^{2}\right)+q_{\mathrm{Fe} X} s_{\mathrm{Fe} X} I_{G}\left[N_{X}+N_{A}\right. \\
& \left.-2\left(q_{\mathrm{Fe} X} s_{\mathrm{Fe} X} I_{G} N_{X} N_{A}\right) t\right]\left(D t+E t^{2}\right) \\
& -q_{X, \mathrm{IR}} s_{X \mathrm{IR}} I_{\mathrm{IR} 1}\left(q_{\mathrm{Fe} X} s_{\mathrm{Fe} X} I_{G} N_{X} N_{A}\right) t .
\end{aligned}
$$

Equating the coefficients of the first two powers of $t$ ( $\mathrm{dc}$ and linear terms) on the two sides of Eqs. (40) and (41), we can find a set of four equations for four unknowns, $B, C, D$, and $E$. Solving such a set of equations results in

$$
\begin{gathered}
B=-\left(\frac{e \mu n_{00}}{\epsilon \epsilon_{0}}+\frac{i K}{e} \kappa_{\mathrm{Fe}} I_{G}\right) A \\
C=\frac{1}{2}\left[\left(\frac{e \mu n_{00}}{\epsilon \epsilon_{0}}+\frac{i K}{e} \kappa_{\mathrm{Fe}} I_{G}\right)^{2}-\frac{e \mu n_{01}}{\epsilon \epsilon_{0}}\right] A \\
-\frac{1}{2}\left[q_{\mathrm{Fe} X} s_{\mathrm{Fe} X} I_{G} N_{X}\left(\frac{i K}{e}\right)\left(\kappa_{X, G} I_{G}+\kappa_{X, \mathrm{IR}} I_{\mathrm{IR} 0}-\kappa_{\mathrm{Fe}} I_{G}\right)\right] A \\
-\frac{i K}{2 e} \kappa_{X, \mathrm{IR}} q_{\mathrm{Fe} X} s_{\mathrm{Fe} X} I_{G} N_{X} N_{A} I_{\mathrm{IR} 1},
\end{gathered}
$$




$$
\begin{gathered}
D=\left(q_{\mathrm{Fe} X} s_{\mathrm{Fe} X} I_{G} N_{X}\right) A \\
E=-\frac{1}{2} q_{\mathrm{Fe} X} s_{\mathrm{Fe} X} I_{G} N_{X}\left(q_{X, G} s_{X, G} I_{G}+q_{X, \mathrm{IR}} s_{X, \mathrm{IR}} I_{\mathrm{IR} 0}\right) A \\
-\frac{1}{2} q_{\mathrm{Fe} X} s_{\mathrm{Fe} X} I_{G} N_{X}\left[q_{\mathrm{Fe} X} s_{\mathrm{Fe} X} I_{G}\left(N_{X}+2 N_{A}\right)+\frac{e \mu n_{00}}{\epsilon \epsilon_{0}}\right. \\
\left.+\frac{i K}{e} \kappa_{\mathrm{Fe}} I_{G}\right] A-\frac{1}{2} q_{X, \mathrm{IR}} s_{X, \mathrm{IR}} q_{\mathrm{Fe} X} s_{\mathrm{Fe} X} I_{G} N_{X} N_{A} I_{\mathrm{IR} 1}
\end{gathered}
$$

\section{E. Saturation space-charge field}

The space-charge field $E_{1}$ within one pulse can be represented as

$$
E_{1}=\frac{-i e}{K \epsilon \epsilon_{0}}\left(A+B t+C t^{2}\right)
$$

with $A, B, C$ defined above. The saturation space-charge field can be easily obtained from Eq. (46) by noting that the space-charge field at the beginning and at the end of each pulse would be the same at saturation. This can be written mathematically as

$$
E_{1}\left(t=t_{p}\right)=E_{1}(t=0)=\frac{-i e}{K \epsilon \epsilon_{0}} A,
$$

or

$$
B+C t_{p}=0
$$

where $t_{p}$ is the pulse width. Putting $B$ and $C$ from Eqs. (42) and (43) into Eq. (48), we can solve for the saturation spacecharge field $\left[-i e A /\left(K \epsilon \epsilon_{0}\right)\right]$ as

$$
\left.E_{1}\right|_{\text {saturation }}=\frac{\beta_{1} I_{\mathrm{IR} 1}}{\beta_{2}+\beta_{3} I_{\mathrm{IR} 0}+\beta_{4} I_{G}}
$$

where

$$
\begin{aligned}
& \beta_{1}=-\frac{t_{p}}{2 \epsilon \epsilon_{0}} q_{\mathrm{Fe} X} S_{\mathrm{Fe} X} N_{A} \kappa_{X, \mathrm{IR}} \\
& \beta_{2}=\frac{e \mu N_{A}}{\epsilon \epsilon_{0} \gamma_{\mathrm{Fe}}\left(N_{\mathrm{Fe}}-N_{A}\right)} q_{\mathrm{Fe}} s_{\mathrm{Fe}}+\frac{i K}{e} \kappa_{\mathrm{Fe}}, \\
& \beta_{3}=\frac{t_{p}}{2} q_{\mathrm{Fe} X} s_{\mathrm{Fe} X} N_{X}\left[\frac{e \mu N_{A}}{\epsilon \epsilon_{0} \gamma_{\mathrm{Fe}}\left(N_{\mathrm{Fe}}-N_{A}\right.}\right) q_{X, \mathrm{IR}} s_{X, \mathrm{IR}}+\frac{i K}{e} \kappa_{X, \mathrm{IR}}, \\
& \beta_{4}=\frac{t_{p}}{2} q_{\mathrm{Fe} X} s_{\mathrm{Fe} X} N_{X}\left[\frac{e \mu N_{A}}{\epsilon \epsilon_{0} \gamma_{\mathrm{Fe}}\left(N_{\mathrm{Fe}}-N_{A}\right)}\right. \\
& \left.\times\left(q_{X, G} s_{X, G}-\frac{N_{\mathrm{Fe}}}{N_{\mathrm{Fe}}-N_{A}} q_{\mathrm{Fe}} S_{\mathrm{Fe}}\right)+\frac{i K}{e}\left(\kappa_{X, G}-\kappa_{\mathrm{Fe}}\right)\right] \\
& -\frac{t_{p}}{2}\left(\frac{e \mu N_{A}}{\epsilon \epsilon_{0} \gamma_{\mathrm{Fe}}\left(N_{\mathrm{Fe}}-N_{A}\right)} q_{\mathrm{Fe}} s_{\mathrm{Fe}}+\frac{i K}{e} \kappa_{\mathrm{Fe}}\right)^{2} \text {. }
\end{aligned}
$$

Equation (49) clearly shows the dependence of the saturation space-charge field (and therefore, saturation hologram strength) on the sensitizing and recording intensities. Later, we will use Eq. (49) to explain the experimental results on the intensity dependence of saturation hologram strength.

\section{F. Time dependence of space-charge field}

In the previous calculations, we solved for the spacecharge field within one pulse. Due to the short lifetime of electrons in the shallow traps, compared to the time between adjacent pulses, we can assume that all electrons in shallow traps at the end of each pulse are transferred locally to the deep traps before the beginning of the next pulse. The local transfer of electrons between traps is based on the fact that almost all electrons are transferred directly from the shallow traps to the deep traps without passing through the conduction band.

To find the dynamics of space-charge formation, we need to calculate the space-charge field in the time scale much longer than one pulse. To avoid confusion, we represent the space-charge field in this time scale by $\overline{E_{1}}$. The change in the space-charge field within one pulse is

$$
\Delta \overline{E_{1}}=E_{1}\left(t=t_{p}\right)-E_{1}(t=0)=-\frac{i e}{K \epsilon \epsilon_{0}}\left(B t_{p}+C t_{p}^{2}\right),
$$

with $t_{p}$ being the pulse width. Therefore, we can write an approximate equation for $\overline{E_{1}}$ as

$$
\frac{d \overline{E_{1}}}{d t} \simeq \frac{\Delta \overline{E_{1}}}{t_{p}}=-\frac{i e}{K \epsilon \epsilon_{0}}\left(B+C t_{p}\right) .
$$

Note that $B$ and $C$ in Eq. (55) are now time dependent, as they are different within different pulses. Replacing $B$ and $C$ from Eqs. (42) and (43) into Eq. (55), we obtain

$$
\begin{aligned}
\frac{d \overline{E_{1}}}{d t}= & \left\{-\left(\frac{e \mu n_{00}}{\epsilon \epsilon_{0}}+\frac{i K}{e} \kappa_{\mathrm{Fe}} I_{G}\right)\right. \\
& \left.+\frac{t_{p}}{2}\left[\left(\frac{e \mu n_{00}}{\epsilon \epsilon_{0}}+\frac{i K}{e} \kappa_{\mathrm{Fe}} I_{G}\right)^{2}-\frac{e \mu n_{01}}{\epsilon \epsilon_{0}}\right]\right\}\left(-\frac{i e}{K \epsilon \epsilon_{0}} A\right) \\
& -\frac{t_{p}}{2} q_{\mathrm{Fe} X} s_{\mathrm{Fe} X} I_{G} N_{X}\left(\frac{i K}{e}\right)\left(\kappa_{X, G} I_{G}+\kappa_{X, \mathrm{IR}} I_{\mathrm{IR} 0}-\kappa_{\mathrm{Fe}} I_{G}\right) \\
& \times\left(-\frac{i e}{K \epsilon \epsilon_{0}} A\right)-\left(\frac{t_{p}}{2 \epsilon \epsilon_{0}} \kappa_{X, \mathrm{IR}} q_{\mathrm{Fe} X} s_{\mathrm{Fe} X} I_{G} N_{X} N_{A}\right) I_{\mathrm{IR} 1} .
\end{aligned}
$$

Note that $-i e A /\left(K \epsilon \epsilon_{0}\right)$ is the space-charge field at the beginning of each pulse, and therefore we can write

$$
\overline{E_{1}}(t) \simeq-\frac{i e}{K \epsilon \epsilon_{0}} A(t)
$$

Combining Eqs. (56) and (57), we obtain 


$$
\frac{d \overline{E_{1}}}{d t}=-\frac{\overline{E_{1}}}{\tau_{r}}+\frac{\left.E_{1}\right|_{\text {saturation }}}{\tau_{r}},
$$

where the saturation space-charge field $\left.E_{1}\right|_{\text {saturation }}$ is the same as that obtained by a simple observation previously [Eq. (49)], and recording speed (inverse of recording time constant $\tau_{r}$ ) is given by

$$
\begin{aligned}
\frac{1}{\tau_{r}}= & \left(\frac{e \mu n_{00}}{\epsilon \epsilon_{0}}+\frac{i K}{e} \kappa_{\mathrm{Fe}} I_{G}\right)-\frac{t_{p}}{2}\left(\frac{e \mu n_{00}}{\epsilon \epsilon_{0}}+\frac{i K}{e} \kappa_{\mathrm{Fe}} I_{G}\right)^{2} \\
& +\frac{t_{p}}{2}\left[\frac{e \mu n_{01}}{\epsilon \epsilon_{0}}+q_{\mathrm{Fe} X} s_{\mathrm{Fe} X} I_{G} N_{X}\left(\frac{i K}{e}\right)\left(\kappa_{X, G} I_{G}+\kappa_{X, \mathrm{IR}} I_{\mathrm{IR} 0}-\kappa_{\mathrm{Fe}} I_{G}\right)\right] \\
= & \left(\frac{e \mu}{\epsilon \epsilon_{0}} \frac{q_{\mathrm{Fe}} s_{\mathrm{Fe}} N_{A}}{\gamma_{\mathrm{Fe}}\left(N_{\mathrm{Fe}}-N_{A}\right)}+\frac{i K}{e} \kappa_{\mathrm{Fe}}\right) I_{G}-\frac{t_{p}}{2}\left(\frac{e \mu}{\epsilon \epsilon_{0}} \frac{q_{\mathrm{Fe}} s_{\mathrm{Fe}} N_{A}}{\gamma_{\mathrm{Fe}}\left(N_{\mathrm{Fe}}-N_{A}\right)}+\frac{i K}{e} \kappa_{\mathrm{Fe}}\right)^{2} I_{G}^{2}+\frac{t_{p}}{2} q_{\mathrm{Fe} X} s_{\mathrm{Fe} X} N_{X}\left(\frac{e \mu}{\epsilon \epsilon_{0}} \frac{N_{A}}{\gamma_{\mathrm{Fe}}\left(N_{\mathrm{Fe}}-N_{A}\right)}\right) \\
& \times\left(q_{X, G} s_{X, G}-\frac{N_{\mathrm{Fe}}}{N_{\mathrm{Fe}}-N_{A}} q_{\mathrm{Fe}} s_{\mathrm{Fe}}\right) I_{G}^{2}+\frac{t_{p}}{2} q_{\mathrm{Fe} X} s_{\mathrm{Fe} X} N_{X}\left(\frac{i K}{e}\right)\left(\kappa_{X, G}-\kappa_{\mathrm{Fe}}\right) I_{G}^{2}+\frac{t_{p}}{2} q_{\mathrm{Fe} X} s_{\mathrm{Fe} X} N_{X}\left(\frac{e \mu}{\epsilon \epsilon_{0}} \frac{N_{A}}{\gamma_{\mathrm{Fe}}\left(N_{\mathrm{Fe}}-N_{A}\right)}\right) \\
& \times\left(q_{X, \mathrm{IR}} s_{X, \mathrm{IR}}+\frac{i K}{e} \kappa_{X, \mathrm{IR}}\right) I_{G} I_{\mathrm{IR} 0} .
\end{aligned}
$$

The solution of Eq. (58) for $\overline{E_{1}}$ with initial condition $\overline{E_{1}}(t=0)=0$ is a monoexponential function like

$$
\overline{E_{1}}=\left.E_{1}\right|_{\text {saturation }}\left[1-\exp \left(-\frac{t}{\tau_{r}}\right)\right] \text {. }
$$

This formula does not show the variation of the space-charge field within the individual pulses. This is acceptable, since in the experiments we measure the diffraction efficiency of the holograms after pulses and not within them. Note that the time variable $t$ in Eq. (60) is the time where the pulse is on (exposure time). The space-charge field remains constant between adjacent pulses. Therefore, we delete the times when the pulse is off from the time variable $t$. Note that Eq. (58) can also be used with a different initial condition to obtain the space-charge field during erasure. Therefore, the recording and erasure time constants are equal. In Sec. VI we will use Eqs. (49) and (59) to explain the experimental dependence of the saturation space-charge field and recording time constant on the intensities of the sensitizing and recording beams.

We can improve the accuracy of the analytical formula derived above by using more terms in the Taylor-series expansion of different variables. The next approximation step is to consider the first three Taylor-series terms for the zeroorder variables and the first four ones for the first-order variables.

\section{G. Simplified formulas}

Although we derived analytic formulas for the saturation space-charge field and recording time constant [Eqs. (49) and (59), respectively], the formulas are so complex that we cannot easily use them to explain the different experimental observations based on the simple physical mechanisms. In this section, we use the parameter values from Table I to calcu- late the order of magnitude of the different terms in Eqs. (49) and (59). We then neglect the terms that are at least one order of magnitude less than the others to obtain simplified formulas. In these calculations, we assume $I_{G} \sim I_{\mathrm{IR} 0} \sim 10^{7}$ $-10^{8} \mathrm{~W} / \mathrm{cm}^{2}$ for the sensitizing and recording intensities, $t_{p} \simeq 5 \mathrm{nsec}$ for the pulse width, and $\Lambda \simeq 2 \mu \mathrm{m}$ for the grating period at recording wavelength of $\lambda=1064 \mu \mathrm{m}$. We also assume that the oxidation/reduction state of the crystal is such that $N_{A} / N_{\mathrm{Fe}} \sim 0.1$, i.e., about $10 \%$ of the Fe traps are initially occupied by electrons. These are typical values used in the experiments.

\section{Simplified formula for saturation space-charge field}

Using material parameter values from Table I and experimental values given above, we can simplify Eq. (50) by using the following approximations:

$$
\begin{gathered}
\frac{K}{e}\left|\kappa_{\mathrm{Fe}}\right| \ll \frac{e \mu N_{A}}{\epsilon \epsilon_{0} \gamma_{\mathrm{Fe}}\left(N_{\mathrm{Fe}}-N_{A}\right)} q_{\mathrm{Fe}} s_{\mathrm{Fe}}, \\
\frac{K}{e}\left|\kappa_{X, \mathrm{IR}}\right| \ll \frac{e \mu N_{A}}{\epsilon \epsilon_{0} \gamma_{\mathrm{Fe}}\left(N_{\mathrm{Fe}}-N_{A}\right)} q_{X, \mathrm{IR}} s_{X, \mathrm{IR}},
\end{gathered}
$$

$$
\begin{aligned}
\frac{K}{e}\left|\kappa_{X, G}-\kappa_{\mathrm{Fe}}\right| \ll & \frac{e \mu N_{A}}{\epsilon \epsilon_{0} \gamma_{\mathrm{Fe}}\left(N_{\mathrm{Fe}}-N_{A}\right)} \\
& \times\left|q_{X, G} s_{X, G}-\frac{N_{\mathrm{Fe}}}{\left(N_{\mathrm{Fe}}-N_{A}\right)} q_{\mathrm{Fe}} s_{\mathrm{Fe}}\right|,
\end{aligned}
$$




$$
\begin{aligned}
& \frac{e \mu N_{A}}{\epsilon \epsilon_{0} \gamma_{\mathrm{Fe}}\left(N_{\mathrm{Fe}}-N_{A}\right)}\left(q_{\mathrm{Fe}} s_{\mathrm{Fe}}\right)^{2} \\
& \quad \ll q_{\mathrm{Fe} X} s_{\mathrm{Fe} X} N_{X}\left|q_{X, G} s_{X, G}-\frac{N_{\mathrm{Fe}}}{N_{\mathrm{Fe}}-N_{A}} q_{\mathrm{Fe}} s_{\mathrm{Fe}}\right|,
\end{aligned}
$$

where Eq. (61) is used for simplification of $\beta_{2}$ in Eq. (51); Eq. (62) is used for simplification of $\beta_{3}$ in Eq. (51); and Eqs. (63) and (64) are used for simplification of $\beta_{4}$. For the crystal used in our experiments, the right-hand-sides of Eqs. (61)-(63) are larger than their corresponding left-hand sides by at least a factor of 200. This factor is 40 for Eq. (64). Using these approximations, the simplified formula for the saturation space-charge field $\left.E_{1}\right|_{\text {saturation }}$ becomes

$$
\left.E_{1}\right|_{\text {saturation }}=\frac{-\frac{t_{p}}{2} q_{\mathrm{FeX}} s_{\mathrm{Fe} X} N_{A} N_{X} \kappa_{X, \mathrm{IR}} I_{\mathrm{IR} 1}}{\frac{e \mu N_{A}}{\gamma_{\mathrm{Fe}}\left(N_{\mathrm{Fe}}-N_{A}\right)} \mathcal{T}}
$$

where

$$
\begin{aligned}
\mathcal{T}= & \frac{t_{p}}{2} q_{\mathrm{Fe} X} s_{\mathrm{Fe} X} N_{X}\left[q_{X, \mathrm{IR}} s_{X, \mathrm{IR}} I_{\mathrm{IR} 0}\right. \\
& \left.+\left(q_{X, G} s_{X, G}-\frac{N_{\mathrm{Fe}}}{\left(N_{\mathrm{Fe}}-N_{A}\right)} q_{\mathrm{Fe}} s_{\mathrm{Fe}}\right) I_{G}\right]+q_{\mathrm{Fe}} s_{\mathrm{Fe}}
\end{aligned}
$$

Equation (65) can be rewritten in a form that is very useful for understanding the main physical mechanisms responsible for recording by multiplying the numerator and the denomi-

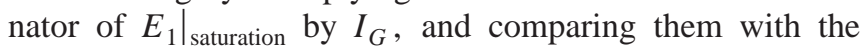
values of $n_{0}$ and $N_{X 0}^{-}$averaged over one pulse width $(0 \leqslant t$ $\leqslant t_{p}$ ) given below by $n_{0 \text {,ave }}$ and $N_{X 0 \text {,ave }}^{-}$,

$$
\begin{aligned}
n_{0, \mathrm{ave}}= & n_{00}+n_{01} \frac{t_{p}}{2} \\
= & \frac{N_{A}}{\gamma_{\mathrm{Fe}}\left(N_{\mathrm{Fe}}-N_{A}\right)} \frac{t_{p}}{2} q_{\mathrm{Fe} X} s_{\mathrm{Fe} X} N_{X} \\
& \times\left(q_{X, G} s_{X, G}-\frac{N_{\mathrm{Fe}}}{\left(N_{\mathrm{Fe}}-N_{A}\right)} q_{\mathrm{Fe}} s_{\mathrm{Fe}}\right) I_{G}^{2} \\
& +\frac{N_{A}}{\gamma_{\mathrm{Fe}}\left(N_{\mathrm{Fe}}-N_{A}\right)} \\
& \times\left(q_{\mathrm{Fe}} s_{\mathrm{Fe}}+\frac{t_{p}}{2} q_{\mathrm{Fe} X} s_{\mathrm{Fe} X} N_{X} q_{X, \mathrm{IR}} s_{X, \mathrm{IR}} I_{\mathrm{IR} 0}\right) I_{G}, \\
& N_{X 0, \mathrm{ave}}^{-}=\frac{t_{p}}{2} q_{\mathrm{Fe} X} s_{\mathrm{Fe} X} N_{X} N_{A} I_{G} .
\end{aligned}
$$

The resulting simplified formula for the saturation spacecharge field is

$$
\left.E_{1}\right|_{\text {saturation }}=-\frac{\kappa_{X, \mathrm{IR}} N_{X 0, \mathrm{ave}}^{-} I_{\mathrm{IR} 1}}{e \mu n_{0, \mathrm{ave}}} .
$$

In the next section, we will use this formula to explain different experimental observations based on very basic physical mechanisms.

\section{Simplified formula for recording time constant}

Using the approximations given by Eqs. (61)-(64), we can simplify Eq. (59) for the recording speed as

$$
\begin{aligned}
\frac{1}{\tau_{r}}= & \frac{1}{t_{p}}\left[\frac { e \mu } { 2 \epsilon \epsilon _ { 0 } } \frac { N _ { A } } { \gamma _ { \mathrm { Fe } } ( N _ { \mathrm { Fe } } - N _ { A } ) } q _ { \mathrm { Fe } X } s _ { \mathrm { Fe } X } N _ { X } \left(q_{X, G} s_{X, G}\right.\right. \\
& \left.-\frac{N_{\mathrm{Fe}}}{N_{\mathrm{Fe}}-N_{A}} q_{\mathrm{Fe}} s_{\mathrm{Fe}}\right)\left(I_{G} t_{p}\right)^{2}+\frac{e \mu}{\epsilon \epsilon_{0}} \frac{N_{A}}{\left(N_{\mathrm{Fe}}-N_{A}\right)}\left(q_{\mathrm{Fe}} s_{\mathrm{Fe}}\right. \\
& \left.\left.+\frac{1}{2} q_{\mathrm{Fe} X} s_{\mathrm{Fe} X} N_{X} q_{X, \mathrm{IR}} s_{X, \mathrm{IR}}\left(I_{\mathrm{IR} 0} t_{p}\right)\right)\left(I_{G} t_{p}\right)\right]
\end{aligned}
$$

Comparing Eqs. (70) and (67), we obtain the following simple formula that can be used to explain the experimental observations based on simple physical mechanisms

$$
\frac{1}{\tau_{r}}=\frac{e \mu n_{0, \mathrm{ave}}}{\epsilon \epsilon_{0}} .
$$

\section{H. Comparison with numerical solution}

Figures 11(a) and 11(b) show the variations of saturation change in the index of refraction $\Delta n=$ $-\left.\left(n^{3} / 2\right) r_{13} E_{1}\right|_{\text {saturation }}(n$, index of refraction at recording frequency) with recording and sensitizing intensities ( $I_{\text {IR0 }}$ and $\left.I_{G}\right)$, respectively. In these figures, we have shown both analytical and numerical solutions as well as the experimental results. As Fig. 11 shows, the agreement between the analytical formula for $\left.E_{1}\right|_{\text {saturation }}[\mathrm{Eq} .(65)]$ and the numerical solution is very good with all levels of assumptions and approximations involved.

Figures 12(a) and 12(b) show the variations of recording speed $\left(1 / \tau_{r}\right)$ with recording and sensitizing intensities, respectively. As in Fig. 11, we have shown analytical and numerical solutions as well as the experimental results. Although the analytic solution from Eq. (70) shows the appropriate qualitative variation of recording speed with intensities, its deviation from the numerical solution is more than $10 \%$ for larger intensities, as shown in Fig. 12. One of the major sources of error in the analytic solution is the approximation $n_{0} \simeq n_{00}+n_{01} t$ given by Eq. (29). To obtain a more accurate formula for the recording speed, we use the simplified formula given by Eq. (71), but we calculate $n_{0 \text {,ave }}$ by time averaging $n_{0}$ without making a linear approximation. To do this, we first replace the more accurate formula for $N_{X 0}^{-}$from Eq. (24) into the formula for $n_{0}$ given by Eq. (29) and rearrange the terms to obtain

$$
n_{0}=\frac{\zeta_{1}-\zeta_{2} \exp \left(-t / \tau_{x}\right)}{\zeta_{3}-\zeta_{4} \exp \left(-t / \tau_{x}\right)},
$$


(a)

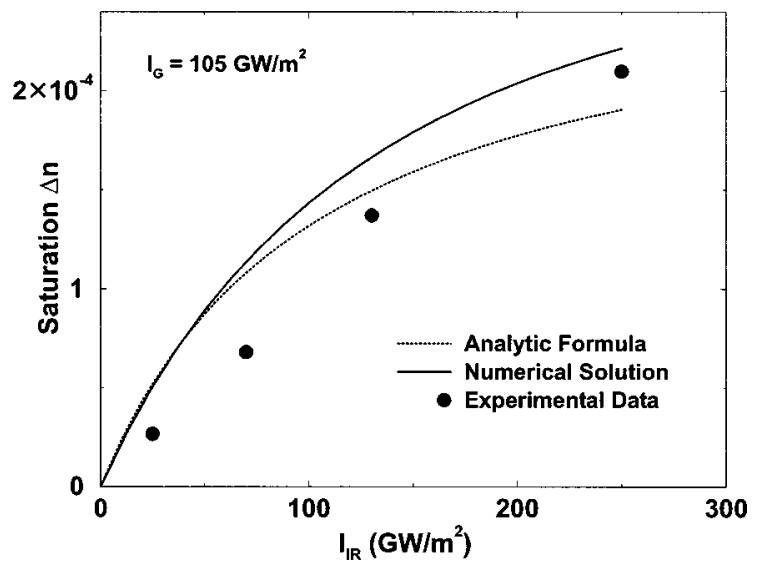

(b)

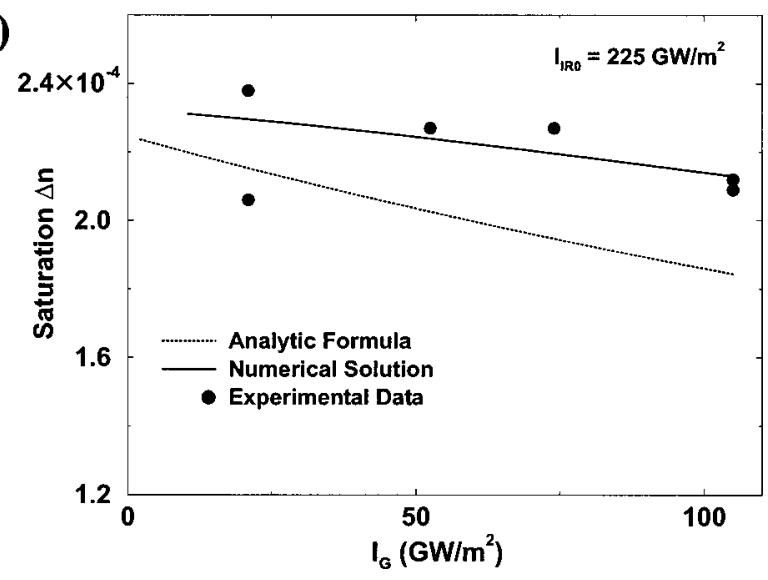

FIG. 11. Variation of the saturation value of the amplitude of the refractive index grating $(\Delta n)$ with (a) average infrared light intensity $I_{\mathrm{IR} 0}$ while green light intensity is fixed $\left(I_{G}\right.$ $=105 \mathrm{GW} / \mathrm{m}^{2}$ ), and (b) green light intensity $I_{G}$ while infrared light intensity is fixed $\left(I_{\mathrm{IR} 0}=225 \mathrm{GW} / \mathrm{m}^{2}\right)$. The modulation depth of the infrared intensity was $1\left(I_{\mathrm{IR} 0}=I_{\mathrm{IR} 1}\right)$ in both cases.

where, $\tau_{x}$ is given by Eq. (25), and $\zeta_{1}-\zeta_{4}$ are defined by

$$
\begin{aligned}
\zeta_{1}= & q_{\mathrm{Fe}} s_{\mathrm{Fe}} I_{G} N_{A}\left(q_{X, G} s_{X, G} I_{G}+q_{X, \mathrm{IR}} s_{X, \mathrm{IR}} I_{\mathrm{IR} 0}\right. \\
& \left.+q_{\mathrm{Fe} X} s_{\mathrm{Fe} X} I_{G} N_{A}\right) \\
& +q_{\mathrm{Fe} X} s_{\mathrm{Fe} X} I_{G} N_{X} N_{A}\left(q_{X, G} s_{X, G} I_{G}+q_{X, \mathrm{IR}} s_{X, \mathrm{IR}} I_{\mathrm{IR} 0}\right),
\end{aligned}
$$

$$
\begin{aligned}
\zeta_{2}= & q_{\mathrm{Fe} X} s_{\mathrm{Fe} X} I_{G} N_{X} N_{A}\left(q_{X, G} s_{X, G} I_{G}+q_{X, \mathrm{IR}} s_{X, \mathrm{IR}} I_{\mathrm{IR} 0}\right. \\
& \left.-q_{\mathrm{Fe}} s_{\mathrm{Fe}} I_{G}\right), \\
\zeta_{3}= & \gamma_{\mathrm{Fe}}\left[( N _ { \mathrm { Fe } } - N _ { A } ) \left(q_{X, G} s_{X, G} I_{G}+q_{X, \mathrm{IR}} s_{X, \mathrm{IR}} I_{\mathrm{IR} 0}\right.\right. \\
+ & \left.\left.q_{\mathrm{Fe} X} s_{\mathrm{Fe} X} I_{G}\left[N_{X}+N_{A}\right]\right)\right]+\gamma_{\mathrm{Fe}} q_{\mathrm{Fe} X} s_{\mathrm{Fe} X} I_{G} N_{X} N_{A},
\end{aligned}
$$

$$
\zeta_{4}=\gamma_{\mathrm{Fe}} q_{\mathrm{Fe} X} s_{\mathrm{Fe} X} I_{G} N_{X} N_{A}
$$

In the next step, we calculate $n_{0 \text {,ave }}$ by time-averaging $n_{0}$ from Eq. (72) over one pulse width $\left(0 \leqslant t \leqslant t_{p}\right)$, (a)
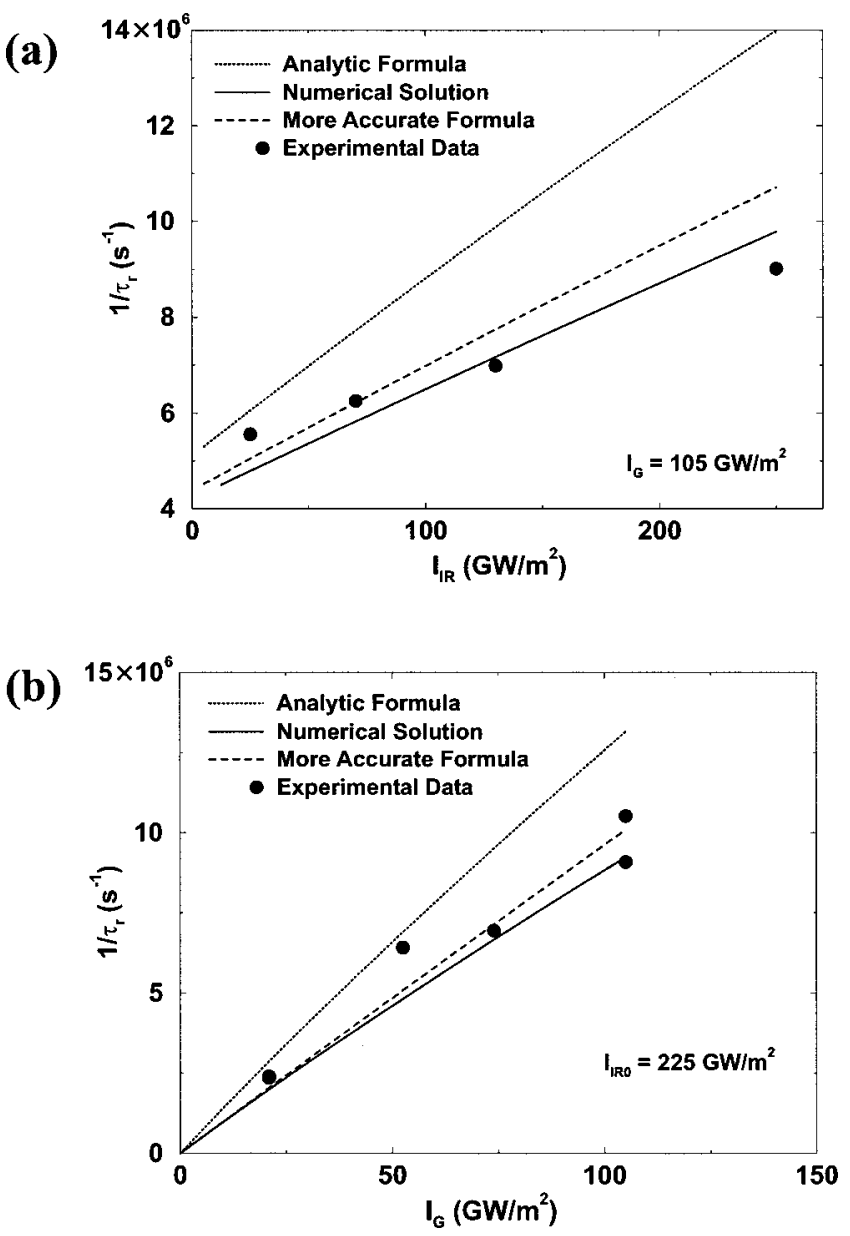

FIG. 12. Variation of recording speed $\left(\tau_{r}^{-1}\right)$ with (a) average infrared light intensity $I_{\text {IR0 }}$ while green light intensity is fixed $\left(I_{G}\right.$ $=105 \mathrm{GW} / \mathrm{m}^{2}$ ), and (b) green light intensity $I_{G}$ while infrared light intensity is fixed $\left(I_{\mathrm{IR} 0}=225 \mathrm{GW} / \mathrm{m}^{2}\right)$. The modulation depth of the infrared intensity was $1\left(I_{\mathrm{IR} 0}=I_{\mathrm{IR} 1}\right)$ in both cases.

$$
\begin{aligned}
n_{0, \mathrm{ave}} & =\frac{1}{t_{p}} \int_{0}^{t_{p}} n_{0} d t=\frac{1}{t_{p}} \int_{0}^{t_{p}} \frac{\zeta_{1}-\zeta_{2} \exp \left(-t / \tau_{x}\right)}{\zeta_{3}-\zeta_{4} \exp \left(-t / \tau_{x}\right)} d t \\
& =\frac{\zeta_{1}}{\zeta_{3}}+\frac{\tau_{x}}{t_{p}}\left(\frac{\zeta_{2}}{\zeta_{4}}-\frac{\zeta_{1}}{\zeta_{3}}\right) \ln \left[\frac{\zeta_{3}-\zeta_{4}}{\zeta_{3}-\zeta_{4} \exp \left(-t_{p} / \tau_{x}\right)}\right] .
\end{aligned}
$$

Putting $n_{0 \text {,ave }}$ into Eq. (71), we obtain a more accurate analytic formula for the recording time constant. The variation of recording speed with sensitizing and recording intensities using this more accurate formula is also depicted in Fig. 12 showing much better agreement with the numerical solution than the approximate formula given by Eq. (70). Therefore, we have analytic formulas for both the saturation spacecharge field and recording time constant that agree very well with both the numerical solution and experimental results.

It is important to note that the analytic formulas become less accurate as we increase either the intensities or the pulse width. This is due to the fact that increasing the energy of each pulse (by increasing either its intensity or its width) results in stronger variation of the variables within one pulse 
and makes the approximation of the variables by a few Taylor-series terms less accurate. However, the analytic formulas derived in this section are good enough for most practical applications with current high-energy pulsed lasers. The more significant usage of these formulas is the understanding of main physical mechanisms responsible for holographic recording and using them for the explanation of the experimental observations. This is done in the next section.

\section{EXPLANATION OF THE EXPERIMENTAL OBSERVATIONS}

In this section, we use the two simplified formulas we derived in the last section to draw a simple physical picture for pulse recording mechanisms and use it to explain the experimental observations discussed in Sec. IV. In this section, we assume that $I_{\mathrm{IR} 0}=I_{\mathrm{IR} 1}$, in agreement with experimental conditions. Therefore, we use $I_{\mathrm{IR} 0}$ when the variation with recording intensity is involved. We repeat the simplified formulas (69) and (71) here:

$$
\begin{aligned}
\left.E_{1}\right|_{\text {saturation }} & =-\frac{\kappa_{X, \mathrm{IR}} N_{X 0, \mathrm{ave}}^{-} I_{\mathrm{IR} 1}}{e \mu n_{0, \mathrm{ave}}}, \\
\frac{1}{\tau_{r}} & =\frac{e \mu n_{0, \mathrm{ave}}}{\epsilon \epsilon_{0}} .
\end{aligned}
$$

The formula for recording speed is similar to that for normal holographic recording with $\mathrm{cw}$ light in singly doped $\mathrm{LiNbO}_{3}$ crystals. The only difference is that in the latter we have the dc electron concentration in the conduction band $\left(n_{0}\right)$ in place of $n_{0 \text {,ave }}$, the time-averaged dc electron concentration in the conduction band over one pulse width. The formula for the saturation space-charge field is also similar to what we have in normal $\mathrm{cw}$ recording. This similarity is better understood by recalling that the total current density $j$ is zero at saturation (steady state). Neglecting diffusion, we can write the above statement mathematically as

$$
\left.j_{1}\right|_{\text {saturation }}=j_{\mathrm{ph} 1}+\left.e \mu n_{0} E_{1}\right|_{\text {saturation }}=0,
$$

or

$$
\left.E_{1}\right|_{\text {saturation }}=-\frac{j_{\mathrm{ph} 1}}{e \mu n_{0}} .
$$

If we assume that the dominant term in the bulk photovoltaic current is that from the shallow traps due to the recording light, we can rewrite Eq. (79) as

$$
\left.E_{1}\right|_{\text {saturation }}=-\frac{\kappa_{X, \mathrm{IR}} N_{X 0}^{-} I_{\mathrm{IR} 0}}{e \mu n_{0}},
$$

where we assumed $I_{\mathrm{IR} 1}=I_{\mathrm{IR} 0}$. Equation (80) becomes the same simplified formula we derived for $\left.E_{1}\right|_{\text {saturation }}$ if we replace $N_{X 0}^{-}$and $n_{0}$ by their time-averaged values over one pulse width, $N_{X 0 \text {,ave }}^{-}$and $n_{0 \text {,ave }}$, respectively.

Although the physical mechanisms in two-step holographic recording with high intensity pulses are similar to those of normal recording, the intensity dependence of the (a)

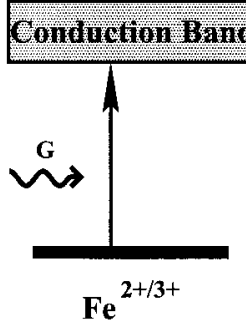

Valence Band (b)

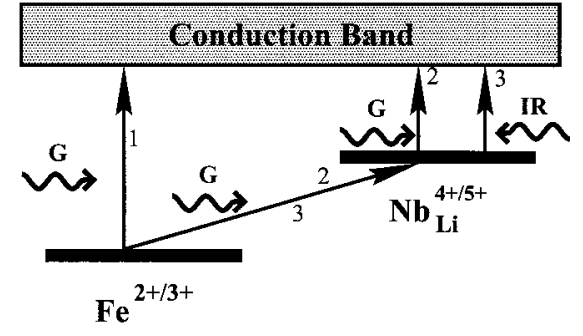

Varience Band

FIG. 13. Mechanisms for excitation of electrons from deep traps to the conduction band in a $\mathrm{LiNbO}_{3}: \mathrm{Fe}$ crystal for (a) normal recording with low intensities, and (b) two-step recording with high intensities. There are three different paths for electron generation in two-step recording indicated by 1,2 , and 3 . In part (b), electron transfer mechanisms caused by sensitizing (green) light are indicated by $G$, and those caused by recording (infrared) light are indicated by IR.

saturation space-charge field and recording speed in the two cases are different. This is due to the fact that the trap responsible for electron concentration in the conduction band, and the one responsible for the bulk photovoltaic current, are the same in normal recording, while they are different in two-step recording. This can be easily understood from Fig. 13 that shows the energy band diagrams of the two cases. In normal recording, the electron concentration in the conduction band is due to excitation from Fe traps by the recording light. The same traps are also responsible for the bulk photovoltaic effect caused by the recording light. Therefore, both $j_{\mathrm{ph} 1}$ and $n_{0}$ in Eq. (79) depend linearly on recording intensity. As a result, the saturation space-charge field in normal recording is independent of recording intensity. On the other hand, recording speed $\left(1 / \tau_{r}\right)$ in normal recording increases linearly with recording intensity since $n_{0}$ has this intensity dependence.

In two-step recording, the electron concentration in the conduction band is caused by three different paths: directly from the deep traps by sensitizing light [path 1 in Fig. 13(b)], in two steps via the shallow traps by sensitizing light only [path 2 in Fig. 13(b)], and from the deep traps to the shallow traps by sensitizing light; then from shallow traps to the conduction band by recording light [path 3 in Fig. 13(b)]. The strengths of these three mechanisms depend on $I_{G}, I_{G}^{2}$, and $I_{G} I_{\mathrm{IR} 0}$, respectively. The time averaging of $n_{0}$ over one pulse does not change this intensity dependence. This explains the experimentally observed dependence of the recording speed on $I_{G}$ and $I_{\text {IR0 }}$ shown in Fig. 12. At lower intensities, electron excitation via path 1 in Figure 13(b) becomes dominant and the recording speed varies linearly with $I_{G}$ while it is weakly dependent on $I_{\mathrm{IR} 0}$. As we increase intensities, the two-step excitation mechanisms [paths 2 and 3 in Fig. 13(b)] become stronger. Therefore, we might observe a quadratic dependence $\left(a_{1} I_{G}+a_{2} I_{G}^{2}\right)$ of the recording speed with $I_{G}$ at very high intensities. We also observe a small linear increase of recording speed with increasing $I_{\mathrm{IR} 0}$ while $I_{G}$ is fixed. During erasure with sensitizing light only, we also observe a quadratic dependence of the erasure speed 
(inverse of erasure time constant) with $I_{G}$ as we have similar dependence of erasure time constant on $n_{0, \text { ave }}$.

As Fig. 11 shows, the saturation hologram strength increases linearly with $I_{\mathrm{IR} 0}$ and decreases very slowly with $I_{G}$. The intensity dependence of the saturation hologram strength in two-step recording (space-charge field or $\Delta n$ ) has been puzzling, as it is very different from normal recording. As a result, there has been no plausible physical explanation of this dependence yet. However, we can easily understand and explain these puzzling observations using our simple model. One important term in the saturation space-charge field is the time-averaged electron concentration in the shallow traps $N_{X 0 \text {,ave }}^{-}$that depends on both populating and depopulating mechanisms. The main populating mechanism is direct electron transfer from the deep traps by sensitizing light, as the trapping of conduction-band electrons by shallow traps can be neglected. The strength of this populating mechanism depends on $I_{G}$. On the other hand, depopulation of the shallow traps within one pulse is due to excitation of the electrons to the conduction band by both sensitizing and recording light. Note that direct electron transfer from shallow traps to deep traps is another depopulating mechanism. However, we neglect this mechanism during one pulse width (a few nanoseconds) due to a much longer lifetime of electrons in the shallow traps (a few milliseconds) as explained before. To summarize, we expect $N_{X 0 \text {,ave }}^{-}$to increase with $I_{G}$ in a complicated way and decrease with increasing $I_{\mathrm{IR} 0}$. With the assumptions and approximations described before, $N_{X 0 \text {,ave }}^{-}$increases linearly with $I_{G}$, while it is independent of $I_{\text {IR0 }}$ (due to the minor role of $I_{\mathrm{IR} 0}$ in depopulation of the shallow traps within one pulse width).

We are now ready to explain the intensity dependence of $\left.E_{1}\right|_{\text {saturation }}$ as we understand the intensity dependence of all terms involved in Eq. (69). We expect $\left.E_{1}\right|_{\text {saturation }}$ to increase linearly with $I_{\mathrm{IR} 0}$ at lower intensities as both $N_{X 0 \text {,ave }}^{-}$and $n_{0, \text { ave }}$ are almost independent of $I_{\mathrm{IR} 0}$ at lower intensities. This dependence on $I_{\text {IR } 0}$ becomes sublinear and finally turns into independence from $I_{\mathrm{IR} 0}$ when we increase $I_{\mathrm{IR} 0}$ without limit while $I_{G}$ is fixed. The latter behavior is due to the linear dependence of $n_{0 \text {,ave }}$ on $I_{\text {IR0 }}$ at higher values of $I_{\text {IR0 }}$. The saturation space-charge field is almost independent of $I_{G}$ at lower intensities due to the approximately linear dependence of both $N_{X 0 \text { ave }}^{-}$and $n_{0 \text {,ave }}$ on $I_{G}$ at lower intensities. The exact dependence on $I_{G}$ is more complicated and depends also on the oxidation/reduction state of the crystal due to a more complicated dependence of $N_{X 0 \text {,ave }}^{-}$and $n_{0 \text {,ave }}$ on $I_{G}$ that becomes more evident at higher intensities. Equation (49) describes a more complete dependence of $\left.E_{1}\right|_{\text {saturation }}$ on sensitizing and recording intensities. It can be seen from this formula that when the oxidation/reduction state of the crystal is such that the coefficient of $I_{G}$ in the denominator of $\left.E_{1}\right|_{\text {saturation }}\left(\beta_{4}\right)$ in Eq. (49) is positive, the saturation spacecharge field decreases with increasing $I_{G}$. When the oxidation/reduction state is such that this coefficient is negative, the saturation space-charge field increases with increasing $I_{G}$ at normal intensities. If we increase $I_{G}$ without limit, the saturation space-charge field will finally decrease with increasing $I_{G}$ regardless of the oxidation state of the crystal, as suggested by Eq. (49). Note that Eq. (49) was derived by assuming that $N_{X 0 \text {,ave }}^{-} \propto I_{G}$, as shown in Eq. (24). Therefore, the exact dependence of $\left.E_{1}\right|_{\text {saturation }}$ on $I_{G}$ is more complicated than was thought previously.

Although we focused above on the dependence of the saturation hologram strength and recording speed on sensitizing and recording intensities, our model can explain the dependence of these two variables on other parameters. For example, we expect the recording speed to depend on $N_{\mathrm{Fe}^{2+}} / N_{\mathrm{Fe}^{2+}}\left[\right.$ or $\left.N_{A} /\left(N_{\mathrm{Fe}}-N_{A}\right)\right]$, since the main source for electron generation in the conduction band is electron concentration in Fe traps $\left(N_{\mathrm{Fe}^{2+}}\right)$, and the main source for electron trapping from the conduction band is the concentration of empty Fe traps $\left(N_{\mathrm{Fe}^{3+}}\right)$. Therefore,

$$
\frac{1}{\tau_{r}} \propto n_{0, \mathrm{ave}} \propto \frac{N_{\mathrm{Fe}^{2+}}}{N_{\mathrm{Fe}^{3+}}} .
$$

We also expect that $N_{X 0 \text {,ave }}^{-} \propto N_{\mathrm{Fe}^{2+}}$ as the shallow traps are populated by direct electron transfer from the deep traps. Putting the dependence of $N_{X 0 \text {,ave }}^{-}$and $n_{0 \text {,ave }}$ into the formula for $\left.E_{1}\right|_{\text {saturation }}$, we obtain

$$
\left.\left.\Delta n\right|_{\text {saturation }} \propto E_{1}\right|_{\text {saturation }} \propto \frac{N_{X 0 \text {,ave }}^{-}}{n_{0, \text { ave }}} \propto N_{\mathrm{Fe}^{3+}},
$$

which is in agreement with the experimental results depicted in Fig. 9.

To summarize, the simple model based on Eqs. (69) and (71) gives us a complete understanding of the physical mechanisms involved in two-step holographic recording with high intensity pulses and helps us understand and explain the experimental observations that were not all explained before.

\section{CONCLUSIONS}

We developed a full numerical solution as well as an approximate analytic solution for two-step holographic recording in $\mathrm{LiNbO}_{3}: \mathrm{Fe}$ crystals. We found the unknown material parameters by fitting the numerical solution to the experimental results. The two important parameters that were unknown so far and found in this work are the bulk photovoltaic coefficient and absorption cross section for the excitation of the electrons from small polarons in $\mathrm{LiNbO}_{3}$ with infrared light (see Table I). The simplified analytic solution we developed agrees very well with the numerical solution for most practical applications. Furthermore, the analytic solution gives us a very good understanding of the physical processes involved. Such a simple model also helps us explain the experimental observations that were not understood before.

Although our method for obtaining an approximate analytic solution was applied to the problem of two-step holographic recording with pulses, the developed strategy can be used in solving a wide variety of problems involving pulses of actions where each pulse is followed by a much longer relaxation time.

\section{ACKNOWLEDGMENTS}

This work was supported by the U.S. Air Force Office of Scientific Research (AFOSR), and by the AF/Rome Laboratory. 
[1] F. H. Mok, Opt. Lett. 18, 915 (1993).

[2] I. McMichael, W. Christian, D. Pletcher, T. Y. Chang, and J. H. Hong, Appl. Opt. 35, 2375 (1996).

[3] J. Ashley, M.-P. Bernal, M. Blaum, G. W. Burr, H. Coufal, R. K. Grygier, H. Günter, J. A. Hoffnagle, C. M. Jefferson, R. M. MacFarlane, B. Marcus, R. M. Shelby, G. T. Sincerbox, and G. Wittmann, Laser Focus World 32, 81 (1996).

[4] J. J. Amodei and D. L. Staebler, Appl. Phys. Lett. 18, 540 (1971).

[5] F. Micheron and G. Bismuth, Appl. Phys. Lett. 20, 79 (1972).

[6] R. A. Rupp, H. C. Külich, U. Schürk, and E. Krätzig, Ferroelectrics 8, 25 (1987).

[7] H. C. Külich, Opt. Commun. 64, 407 (1987).

[8] D. von der Linde, A. M. Glass, and K. F. Rodgers, Appl. Phys. Lett. 25, 155 (1974).

[9] K. Buse, A. Adibi, and D. Psaltis, Nature (London) 393, 665 (1998).

[10] K. Buse, F. Jermann, and E. Krätzig, Ferroelectrics 141, 197 (1993).

[11] K. Buse, F. Jermann, and E. Krätzig, Appl. Phys. A: Solids Surf. 58, 191 (1994).

[12] K. Buse, F. Jermann, and E. Krätzig, Opt. Mater. 4, 237 (1995).

[13] H. Vormann and E. Krätzig, Solid State Commun. 49, 843 (1984).

[14] Y. S. Bai, R. R. Neurgaonkar, and R. Kachru, Opt. Lett. 21, 567 (1996).

[15] Y. S. Bai and R. Kachru, Phys. Rev. Lett. 78, 2944 (1997).

[16] H. Guenther, G. Wittmann, R. M. Macfarlane, and R. R. Neurgaonkar, Opt. Lett. 22, 1305 (1997).

[17] H. Guenther, R. Macfarlane, Y. Furukawa, K. Kitamura, and
R. Neurgaonkar, Appl. Opt. 37, 7611 (1998).

[18] L. Hesselink, S. S. Orlov, A. Liu, A. Akella, D. Lande, and R. R. Neugaonkar, Science 282, 1089 (1998).

[19] N. Iyi, K. Kitamura, F. Izumi, J. K. Yamamoto, T. Hayashi, H. Asano, and S. Kimura, J. Solid State Chem. 101, 340 (1992).

[20] N. Zotov, H. Boysen, J. Schneider, and F. Frey, Mater. Sci. Forum 166-169, 631 (1994).

[21] F. Jermann and J. Otten, J. Opt. Soc. Am. B 10, 2085 (1993).

[22] L. Paraschis, M. C. Bashaw, A. Liu, and L. Hesselink, J. Opt. Soc. Am. B 14, 2670 (1997).

[23] H. Kurz, E. Krätzig, W. Keune, H. Engelmann, U. Gonser, B. Dischler, and A. Räuber, Appl. Phys. 12, 355 (1977).

[24] G. E. Peterson, A. M. Glass, and T. J. Negran, Appl. Phys. Lett. 19, 130 (1971).

[25] H. Kogelnik, Bell Syst. Tech. J. 48, 2909 (1969).

[26] J. Baquedano, M. Carrascosa, L. Arizmendi, and J. M. Cabrera, J. Opt. Soc. Am. B 4, 309 (1987).

[27] N. V. Kukhtarev, Sov. Tech. Phys. Lett. 2, 438 (1976).

[28] N. V. Kukhtarev, V. B. Markov, S. G. Odoulov, M. S. Soskin, and V. L. Vinetskii, Ferroelectrics 22, 949 (1979).

[29] U. van Stevendaal, K. Buse, H. Malz, H. Veenhuis, and E. Krätzig, J. Opt. Soc. Am. B 15, 2868 (1998).

[30] R. T. Smith and F. S. Welsh, J. Appl. Phys. 42, 2219 (1971).

[31] A. Mansingh and A. Dhar, J. Phys. D 18, 2059 (1985).

[32] K. Onuki, N. Uchida, and T. Saku, J. Opt. Soc. Am. 62, 1030 (1972).

[33] G. J. Edwards and M. Lawrence, Opt. Quantum Electron. 16, 373 (1984).

[34] Y. Ohmori, M. Yamaguchi, K. Yoshino, and Y. Inuishi, Jpn. J. Appl. Phys. 15, 2263 (1976). 